\title{
Dynamical mean field theory for oxide heterostructures
}

\author{
O. Janson, Z. Zhong, G. Sangiovanni, and K. Held
}

\begin{abstract}
Transition metal oxide heterostructures often, but by far not always, exhibit strong electronic correlations. State-of-the-art calculations account for these by dynamical mean field theory (DMFT). We discuss the physical situations in which DMFT is needed, not needed, and where it is actually not sufficient. By means of an example, $\mathrm{SrVO}_{3} / \mathrm{SrTiO}_{3}$, we discuss step-by-step and figure-by-figure a density functional theory(DFT)+DMFT calculation. The second part reviews DFT+DMFT calculations for oxide heterostructure focusing on titanates, nickelates, vanadates, and ruthenates.
\end{abstract}

\section{Introduction}

The extraordinary progress to grow heterostructures of transition metal oxides, atomic-layer-by-atomic-layer, cherished hopes of discovering novel physical phenomena that are non-existing in conventional semiconductor heterostructures. Arguably the most remarkable difference and breeding ground for these hopes is the fact that transition metal oxides are often strongly correlated materials. Thence unconventional and unexpected states or colossal responses are imaginable. Hand-inhand with the experimental analysis of prospective correlation phenomena we need a theoretical tool to address electronic correlations in oxide heterostructures. Obvi-

O. Janson and K. Held

Institute for Solid State Physics, TU Wien, 1040 Vienna, Austria

e-mail: heldeifp.tuwien.ac.at

Z. Zhong

Max Planck Institute for Solid State Physics, 70569 Stuttgart, Germany

e-mail: zhong@fkf.mpg.de

G. Sangiovanni

Institut für Theoretische Physik und Astrophysik, Universität Würzburg, Am Hubland, D-97074

Würzburg, Germany 
ously this requires going beyond density functional theory (DFT). It is instead the realm of dynamical mean field theory (DMFT) $[1,2,3]$ and its merger with DFT for real materials calculations $[4,5]$.

Before turning to this method, let us here in the Introduction address the questions: For which oxide heterostructures are electronic correlations important so that DMFT is needed? Actually, for many oxide heterostructures studied experimentally electronic correlations are not that strong. In particular the $\mathrm{LaAlO}_{3} / \mathrm{SrTiO}_{3}$ prototype [6], which has been at the focus of the experimental efforts at the dawn of the research field, is not strongly correlated. The reason for this is that $\mathrm{SrTiO}_{3}$ is a band insulator with empty Ti- $d$ orbitals. Through the polar catastrophe or oxygen defects, these Ti-bands are doped, but only slightly. With only a few charge carriers in the Ti- $d$ orbitals we are far away from an integer filling of the $d$ orbitals, and the $d$ electrons can quite freely move around the interface without often seeing a Ti site already occupied with a $d$-electron and hence prone to a strong Coulomb interaction. Therefore not surprisingly DFT or even a simple tight binding modeling [7] are sufficient to reproduce or predict the angular resolved photoemission spectroscopy (PES, ARPES) spectra $[8,9,10]$. Let us note that there might be localization of (some of) the $d$-electrons at oxygen defects $[11,12,13,14]$. For this localization electronic correlations play a role.

In general, electronic correlations are strong whenever we are at or close to an integer filling of the $d$-orbitals in some of the layers or at some of the sites. In this situation the Coulomb repulsion $U$ is strong and hence important. It suppresses the mobility of the charge carriers, leads to a strongly correlated metal with strong quasiparticle renormalization or even to a Mott insulating state [15]. Correlations may be stronger $[16,17]$ or weaker [18] than in the corresponding bulk state. This kind of physics is included in and described by the local but dynamic DMFT correlations. Another situation where electronic correlations are important is a Hund's metal $[19,20]$ where several $d$ electrons form a local magnetic moment; the Hund's exchange $J$, and not the Coulomb repulsion $U$ plays the decisive role. Here, the effect of electronic correlations is less pronounced in the one-particle spectrum, but strongly reflects in two-particle correlation functions such as e.g. the magnetic susceptibility.

Electronic correlations can give rise to magnetic and/or orbital ordering. Indeed magnetism and possible spintronic applications is one of the prospective advantages of oxide heterostructures. Such an ordering can already be described by the simpler $\mathrm{DFT}+U[21]$ which is a Hartree-Fock treatment and hence does not include genuine correlations. Indeed, a fully polarized ferromagnet is a single Slater determinant and such a ground state is perfectly accounted for in DFT $+U$. What DMFT additionally covers is the correlated paramagnetic state which competes with the magnetic state. This competition is most relevant for the question whether there is ordering or not. Hence DMFT is superior to DFT $+U$ in its predictive power regarding ordering and also allows us to calculate the critical temperature without further approximations or without an adjustment of $U$.

For the PES also the excited states are important even if the ground state is a single Slater determinant. In this respect, DMFT e.g. describes the extra spin-polaron 
peaks [22] in the spectrum of an antiferromagnet which is beyond the Hartree-Fock physics of DFT $+U$. A symmetry broken $\mathrm{DFT}+U$ calculation is often employed for mimicking a localized state also in the paramagnetic phase. An example are the localized states at oxygen vacancies mentioned above which can be described in both, DMFT [13] and DFT $+U$ [23, 24, 14]. For the PES, DFT $+U$ is at least a valid first approximation. Some aspect such as the spin-polaron peaks are missing, but a gap in the spectrum and a localized state can be described this way.

An important aspect of magnetism is the screening of the magnetic moment. Even though we might have a magnetic moment on the femtosecond time scale, this moment and its direction might fluctuate in time [25]. It is screened. Obviously this is an effect of dynamic correlations included in DMFT, but neither in DFT nor $\mathrm{DFT}+U$. This suppression of the long-time magnetic moment is important for magnetism $[19,26]$. Indeed it is one physical reason why there is no magnetism even if it is predicted by DFT which usually underestimates correlations and tendencies towards magnetic ordering in transition metal oxides. Note that $\mathrm{DFT}+U$, on the other hand, grossly overestimates tendencies of magnetic ordering because its only way to avoid the interaction $U$ is ordering. The screening is a further reason why DMFT is more reliable regarding predictions of magnetic ordering. Let us add that experimentally the short-time local moment is discernible in fast, e.g., $\mathrm{x}$-ray absorption, experiments, whereas no moment will be seen in experiments on a longer time scale, e.g., when measuring the magnetic susceptibility.

DMFT includes local dynamic (quantum) correlations, but neglects spatial correlations. Such non-local correlations can be more important at lower temperatures and for lower dimensional systems. Thence they may be also important for oxide heterostructures which are intrinsically two-dimensional. Non-local correlations give rise to additional physical phenomena beyond the realm of DMFT; and one should always be aware of the limitations of the method employed. Physical phenomena that might be relevant for oxide heterostructures and rely on such non-local correlations are: excitons and further vertex corrections to the conductivity such as weak localization, spin fluctuations that might suppress the DMFT-calculated critical temperature for magnetic ordering, and unconventional superconductivity. Often such phenomena can be understood in terms of simple ladder diagrams in orders of $U$, a treatment which is however not sufficient if correlations are truly strong. Cluster [27] and diagrammatic extensions [28, 29, 30, 31, 32] of DMFT which include all the local DMFT correlations but also non-local correlations beyond are a promising way to include such effects. The latter diagrammatic extensions describe a similar physics understood by the aforementioned ladder diagrams at weak coupling, but now for a strongly correlated system including all the DMFT correlations, e.g., the quasiparticle renormalization. These non-local approaches might, in the future, help us understand non-local correlations, but for the time being DMFT is state-of-theart for correlations in oxide heterostructures and it will remain the method of choice whenever local correlations play the dominant role.

In this chapter, we review the DFT+DMFT approach and its application to oxide heterostructures. Section 2 is devoted to methodological aspects. After explaining the advantages of a DFT+DMFT treatment (Section 2.1), we guide the reader 
through the main steps of a DFT+DMFT calculation for a typical correlated heterostructure - a bilayer of $\mathrm{SrVO}_{3}$ on a $\mathrm{SrTiO}_{3}$ substrate (Section 2.2). We also briefly discuss how to DFT+DMFT results can be compared with the experimental spectra (Section 2.3).

Section 3 reviews the results obtained so far by DFT+DMFT for oxide heterostructures, focusing on titanates, nickelates, vanadates and ruthenates. We start in Section 3.1 with titanates, for which electronic reconstruction can lead to a metallic interface but also oxygen vacancies are relevant as a competing mechanism and can give rise to localized states. In Section 3.2 we turn to nickelates which were the first oxide heterostructure studied in DFT+DMFT. Here, heterostructuring might give rise to a cuprate-like Fermi surface or topological states depending on the direction of stacking. Results for vanadates which hint at possible applications of oxide heterostructures as solar cells or as Mott transistors follow in Section 3.3. Section 3.4 is devoted to ruthenates which are arguably most promising for ferromagnetism and spintronic applications. Finally Section 4 summarizes the chapter and provides a brief outlook.

\section{Steps of a DFT+DMFT calculation illustrated by SVO/STO heterostructures}

To illustrate the DFT+DMFT method, we select a numerically tractable, yet instructive correlated compound - the cubic perovskite $\mathrm{SrVO}_{3}$ (Fig. 1). Being a rare example of a metallic $\mathrm{V}^{4+}$ compound, it shows distinct fingerprints of a correlated metal: the pronounced lower Hubbard peak observed in PES [33], the quasiparticle peak seen in ARPES [34], and the upper Hubbard peak in x-ray absorption [35]. From the computational viewpoint, the high crystal symmetry and the $d^{1}$ electronic configuration render $\mathrm{SrVO}_{3}$ a convenient material for testing new numerical techniques.

The strength of electronic correlations can be drastically affected by a dimensional reduction, as it is indeed the case for ultrathin $\mathrm{SrVO}_{3}$ layers grown on $\mathrm{SrTiO}_{3}$. Here, the reduction of the $\mathrm{SrVO}_{3}$ film thickness down to two monolayers leads to a metal-insulator transition [36] and the formation of quantum well states with an anomalous effective mass [8]. In this chapter, we will use such a 2 SVO:4 STO heterostructure, consisting of a $\mathrm{SrVO}_{3}$ bilayer on four $\mathrm{SrTiO}_{3}$ substrate layers, as a model system to guide the reader through the main steps of a DFT+DMFT calculation. 


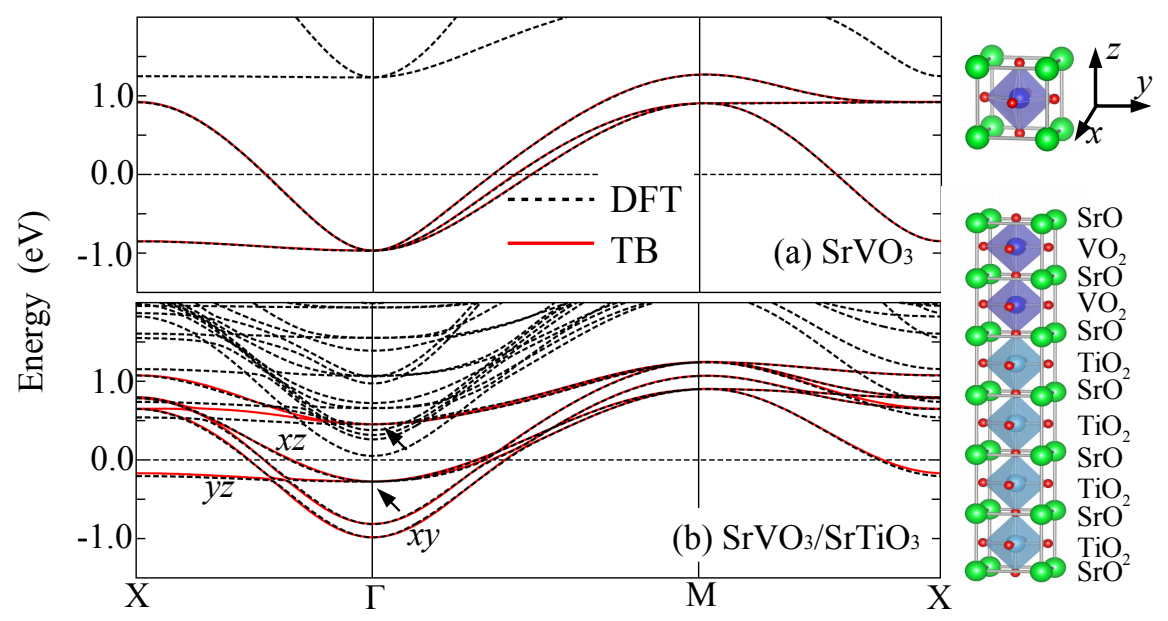

Fig. 1 Left: DFT band structure (black dashed lines) and the $\mathrm{V} t_{2 g}$ bands (red solid lines) calculated for bulk $\mathrm{SrVO}_{3}$. Right top: $\mathrm{SrVO}_{3}$ perovskite cell with $\mathrm{V}$ (blue), $\mathrm{Sr}$ (green) and $\mathrm{O}$ (red) atoms are shown on the right. Right bottom: $\mathrm{SrVO}_{3} / \mathrm{SrTiO}_{3}$ heterostructure containing two $\mathrm{SrVO}_{3}$ layers on a substrate of four $\mathrm{SrTiO}_{3}$ and $10 \AA$ of vacuum. The spatial confinement along $z$ leads to quantized energy levels indicated by arrows for the vanadium $y z / x z$ orbitals at $\Gamma$ in the left panel.

\subsection{Motivation for DFT+DMFT: the electronic structure of bulk $\mathrm{SrVO}_{3}$}

Before introducing the method, we briefly explain the advantages of DFT+DMFT over conventional DFT techniques and DFT $+U$. To this end, we consider bulk $\mathrm{SrVO}_{3} . \mathrm{V}$ atoms in its crystal structure are located in $1 b$ Wyckoff positions with the point group symmetry $m \overline{3} m$. For $d$ electrons $(l=2)$, the irreducible representations are $E_{g}$ (twofold degenerate) and $T_{2 g}$ (threefold degenerate). The electrostatic repulsion between the $\mathrm{V} d$ and $\mathrm{O} p$ electrons (the crystal field) pushes the $e_{g}$ orbitals higher in energy, and the single $d$ electron of $\mathrm{V}$ is distributed over the three degenerate $t_{2 g}$ orbitals.

The DFT band structure of bulk $\mathrm{SrVO}_{3}$ is shown in Fig. 1 (a). At the $\Gamma$ point, the three $t_{2 g}$ bands are degenerate in accord with the space group representation. For an arbitrary $k$-point, e.g. on the $\Gamma$-X $(\pi / a, 0,0)$ path, this degeneracy is partially lifted, and one of the bands (corresponding to the $y z$ orbital along $\Gamma$-X) shows a minute dispersion $(\sim 0.12 \mathrm{eV})$, whereas the two degenerate bands ( $x y$ and $x z$ orbitals) have a sizable dispersion of $\sim 1.9 \mathrm{eV}$ since the orbital lobes are extended in the $x$ direction. If we integrate the DFT bands over the Brillouin zone to obtain the density of states (DOS), it becomes clear that DFT fails to describe the experimental spectral 
features: the DFT DOS in Fig.2 (a) lacks the Hubbard bands, whereas the width of the quasiparticle peak is considerably overestimated (Fig.2, d).

The simplest DFT-based scheme which accounts for on-site interactions in a static mean-field way is DFT $+U$. By construction, this method favors integer orbital occupations [37] and therefore is particularly suitable for orbitally-ordered insulators, but its applicability to correlated metals is at best limited [38]. Indeed, for $\mathrm{SrVO}_{3} \mathrm{DFT}+U$ not only fails to describe the band renormalization, but even yields a spurious magnetic ground state (Fig.2, b).

The idea of DFT $+U$ is to add correlation effects to the DFT on the Hartree-Fock level. In this way, the DFT Hamiltonian is supplemented with a purely real and constant self-energy $\Sigma$ for $\mathrm{V} d$ states. DMFT may be seen as a dynamical version of DFT $+U$, i.e. it accounts also for local scattering processes, and the self-energy $\Sigma$ in DMFT becomes complex and frequency-dependent. This describes (temperaturedependent) scattering processes, which lead to a finite life time. Thus, instead of the DOS, the spectrum of an interacting system is described by the spectral function $A(k, \omega)$. The room-temperature DFT+DMFT spectral function for bulk $\mathrm{SrVO}_{3}$ is shown Fig.2 (c). By comparing it with the experimental spectra Fig.2 (d), we find a considerable improvement over DFT: all spectral features are reproduced, and the width of the quasiparticle peak is in good agreement with the ARPES data.

\subsection{Workflow of a DFT+DMFT calculation}

The main steps of a DFT+DMFT calculation are $^{1}$ : (i) construction of the unit cell, (ii) a DFT calculation, (iii) construction of the low-energy Hamiltonian, (iv) mapping the lattice Hamiltonian onto a set of single-site impurity problems (DMFT), (v) a numerical solution of the resulting single-site models, (vi) adding the doublecounting correction, and (vii) a necessary postprocessing to compute the observables. The way these basic blocks are combined into a computational scheme depends on the implementation and the convergence criterion. In the standard DFT+DMFT scheme, the step (ii) is repeated to converge the electronic density on the DFT level and the steps (v) and (vi) are iterated until the self-energy is converged [5]. In charge-self-consistent calculations, the sequence of steps (ii)-(vi) is repeated until charge redistributions become small [39, 40, 41]. Most computationally demanding schemes involve structural relaxations: the charge redistributions yielded by DMFT are used to calculate forces and provide a new structural input, going back to the step (i) [42]. Such a calculation terminates once the changes in the crystal structure become small enough.

Next, we illustrate all steps in more detail using as an example two $\mathrm{SrVO}_{3}$ layers on top of a $\mathrm{SrTiO}_{3}$ substrate (modeled by four layers), following Ref. [17]. The starting point of a DFT+DMFT calculation is the construction of a unit cell. For (001) heterostructures, it is convenient to think of the $\mathrm{SrVO}_{3}$ perovskite structure as

\footnotetext{
${ }^{1}$ For further details and the theoretical background we refer the reader to [5].
} 

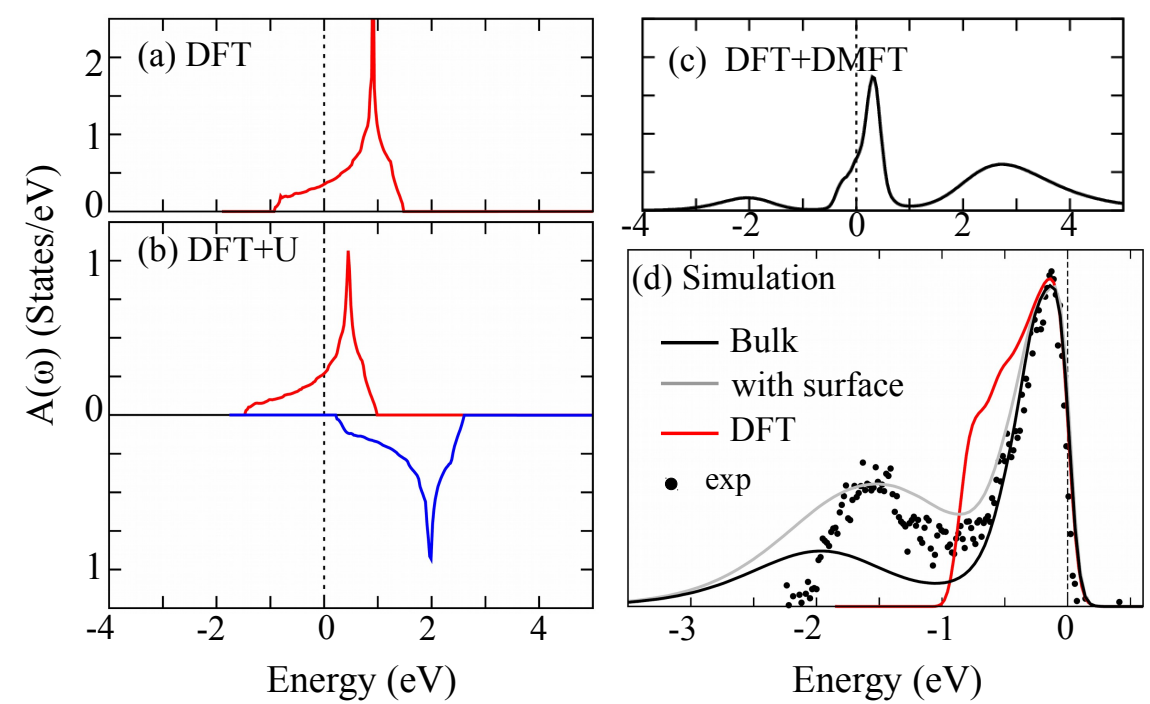

Fig. $2 \mathrm{~V} t_{2 g}$ DOS in (a) DFT yielding a nonmagnetic ground state, (b) DFT $+U\left(U_{d}=2 \mathrm{eV}\right)$ yielding a half-metallic magnetic state with half-filled majority states (red), and (c) DFT+DMFT yielding a nonmagnetic solution with lower Hubbard peak, the upper Hubbard band, and the quasiparticle peak. The DFT+DMFT results show good agreement with the experiment in (d), which can be further improved by including $\mathrm{SrVO}_{3}$ surface spectral contributions (grey).

a periodic alternation of $\mathrm{SrO}$ and $\mathrm{VO}_{2}$ monolayers. By stacking such monolayers on top of each other, we construct a unit cell with two $\mathrm{VO}_{2}$ layers, four $\mathrm{TiO}_{2}$ layers, and seven $\mathrm{SrO}$ layers. Both terminal layers are $\mathrm{SrO}$ monolayers, which ensures that transition metal atoms (V and Ti) have an octahedral coordination. Finally, we make the system effectively two-dimensional by adding a sufficiently thick (10 $⿱$ ) vacuum layer along the $z$ direction, leading to an elongated unit cell shown in the right panel of Fig.1 (b). The lateral lattice constant $a$ of this tetragonal cell is fixed to that of the $\mathrm{SrTiO}_{3}$ substrate $(a=3.92 \AA)$, while all the internal coordinates are optimized in DFT. This latter step is crucial, because surface and strain effects can have a big impact on the crystal structure. For instance, a DFT relaxation of 2 SVO $: 4$ STO yields an inward drift of the surface $\mathrm{Sr}$ atom by $\sim 0.25 \AA$.

The next step is a DFT calculation. Here, we consider the simplest DFT+DMFT scheme: DFT convergence is reached before going to the next step. At present, a plethora of very accurate DFT codes exists [43]; here, we choose the all-electron full potential augmented plane-wave method implemented in Wien2k [44]. It is important to keep in mind that DFT+DMFT results can in some cases sensibly depend on the chosen DFT functional. Most DFT+DMFT studies employ either the local 
Table 1 Leading transfer integrals $t_{i j}\left(\mathbf{R}_{i j}\right)$ (in meV) for bulk SVO and a $2 \mathrm{SVO} / 4 \mathrm{STO}$ heterostructure: onsite terms (first row) and nearest neighbors hopping (along $x$; second row). Due to the mutual orthogonality of $t_{2 g}$ orbitals, only neighboring terms with the same orbital character are nonzero.

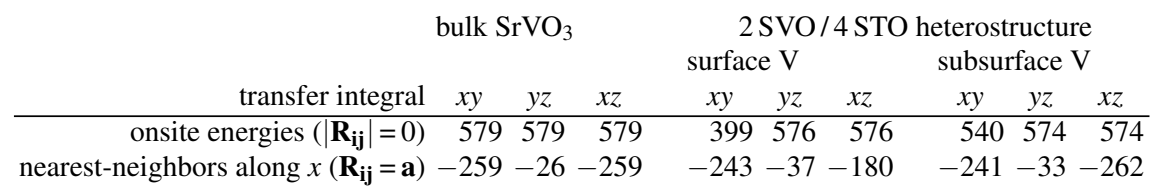

density approximation (LDA) [45, 46] or the generalized gradient approximation (GGA) [47]; here we will use the latter. Finally, DFT calculations are performed on a $k$-mesh, and heterostructures have the advantage of the reduced dimensionality. DFT calculations presented here are performed on a $10 \times 10 \times 1 k$-mesh.

The GGA band structure of the $2 \mathrm{SVO} / 4 \mathrm{STO}$ heterostructure is shown in Fig. 1 (b). The thicket of bands above the Fermi energy are mostly unoccupied Ti $t_{2 g}$ states that will be neglected later in the low-energy model [48]. Moreover, the unit cell has now two inequivalent $\mathrm{VO}_{2}$ monolayers, which doubles the number of $\mathrm{V}$ bands. But the main difference is that the electronic structure becomes two-dimensional: The insulating $\mathrm{SrTiO}_{3}$ substrate on one side and the vacuum on the other side confine the $\mathrm{V} d$ electrons to move in the plane of the bilayer. The partially broken translational symmetry reduces the $\mathrm{V}$ point group to $4 \mathrm{~mm}$, and the $t_{2 g}$ bands at $\Gamma$ split into $a_{1 g}$ $(x y)$ and $e_{g}^{\prime}(y z$ and $x z)$ manifolds. Moreover, the dissimilar potential felt by $\mathrm{V}$ atoms in the surface and subsurface $\mathrm{VO}_{2}$ layers lifts the degeneracy between the $\mathrm{V}$ sites.

DFT results can be used to construct a low-energy model and parameterize its tight-binding part. To this end, the DFT Hamiltonian $H^{\mathrm{DFT}}$ is projected onto a set of localized orbitals. The choice of this correlated subspace depends on the nature of the compound as well as on the problem at hand. For instance, to describe the spectrum of a charge-transfer insulator, one needs to include ligand $p$ states. But in the case of $\mathrm{SrVO}_{3}$, a natural choice for the minimal model is to select the subspace of $\mathrm{V} t_{2 g}$ orbitals. Since there are two inequivalent V atoms in the $2 \mathrm{SVO} / 4 \mathrm{STO}$ heterostructure, the number of orbitals, and hence the dimension of the Hamiltonian matrix are doubled. Using this basis, we search for a tight-binding Hamiltonian $H^{\mathrm{TB}}$, which reproduces the DFT band structure in the corresponding energy range. We therefore construct V-centered maximally localized Wannier orbitals $[49,50,51]$ using the Wannier90 code [52] and the wien2wannier [53] interface which has been integrated into Wien $2 \mathrm{~K}$ recently.

From the maximally localized Wannier functions, we obtain matrix elements of the $6 \times 6 H^{\mathrm{TB}}(\mathbf{k})$ matrix that have the form $\sum_{i j} t_{i j} \exp \left(i \mathbf{R}_{i j} \cdot \mathbf{k}\right)$, where $t_{i j}$ is the transfer integral (hopping) between the orbitals $i$, and $\mathbf{R}_{i j}$ connects their centers. The leading $t_{i j}$ terms are listed in Table 1. The main effect of the confinement is the drastic reduction of the onsite energy for the $\mathrm{V} x y$ orbital of the surface layer, ensuing from the surface reconstruction (the inward drift of surface $\mathrm{Sr}$ atoms).

$H^{\mathrm{TB}}(\mathbf{k})$ describes transfer processes of the low-energy model. In the spirit of the Hubbard model, we supplement this Hamiltonian with an onsite interaction term. In 
the general case, the onsite interaction is described by the interaction vertex $U_{l m n o}$, where $l, m, n$, and $o$ and orbital indices. For practical purposes, simplified versions of this form are typically used [20], e.g. the reduction to the density-density interaction $[l=m$ (same spin), and $n=o$ (same spin)]. Here, we use the rotationally invariant form given by Kanamori $(l=m$ and $n=o$ ), which in addition to densitydensity interactions, accounts accounts for spin exchange and pair hopping processes. The Kanamori Hamiltonian has two parameters: the intra-orbital Coulomb repulsion $U$ and the Hund's exchange $J$, while the inter-orbital Coulomb repulsion $U^{\prime}=U-2 J$ follows from the symmetry. For $\mathrm{V} d$ orbitals, we adopt $U^{\prime}=3.55 \mathrm{eV}$ from constrained LDA calculations $[33,54]$ and $J=0.75 \mathrm{eV}$, which is a standard value for early $3 d$ metal oxides.

The resulting Hamiltonian represents a multi-orbital Hubbard model on a twodimensional lattice. DMFT provides an approximate solution of it, by performing a mapping onto a set of single-site Anderson impurity problems. The unit cells of heterostructures often consist of many (two in the case of $2 \mathrm{SVO} / 4 \mathrm{STO}$ ) correlated atoms, and therefore this mapping involves several steps. First, we denote with $n$ and $m$ the number of atoms in the cell and the number of orbitals per atom, respectively (for the SVO bilayer and $\mathrm{V} t_{2 g}$ orbitals we have $n=2$ and $m=3$ ). Depending on the number and on the type of Wannier functions chosen in the downfolding, the $m$ orbitals associated to each atom can be either considered all as correlated (e.g. the three $t_{2 g}$-orbitals here) or can be further split in a subset of correlated ones and a subset of "ligands" (e.g. p-orbitals). In the latter case the ligands are formally associated to one of the $n$ atoms in the cell, but this is only an arbitrary assignment. In the standard treatment, the interaction term affects only the $d$ correlated orbitals and it is applied locally to each of the $n$ atoms.

We first define the local Green's function $G_{\text {loc }}$ :

$$
G_{\mathrm{loc}}(\omega)=\sum_{\mathbf{k}}\left[(\omega+\mu) \mathbf{I}-H(\mathbf{k})-\Sigma(\omega)-H_{\mathrm{DC}}\right]^{-1},
$$

where $\omega$ is the complex frequency, $\mu$ is the chemical potential, $\mathbf{I}$ is the unitary matrix and $\Sigma(\omega)$ the self energy matrix of dimension $m n \times m n$. The latter is usually set to zero in the first DMFT cycle, and $H_{\mathrm{DC}}$ is the double-counting correction, which will be discussed later.

We now consider the $i$-th atom and start to construct the corresponding impurity problem. The $i$-th $m \times m$ block along the diagonal of $G_{\mathrm{loc}}$ is denoted as $G_{\mathrm{loc}, i}$ $(0<i \leq n)$. The $i$-th impurity Weiss' field $\mathscr{G}_{i}$ is constructed by inverting the corresponding $G_{\mathrm{loc}, i}$ :

$$
\mathscr{G}_{i}(\omega)=\left[\left(G_{\mathrm{loc}, i}\right)^{-1}+\Sigma_{i}(\omega)\right]^{-1},
$$

where $\Sigma_{i}(\omega)$ is now a $m \times m$ diagonal matrix containing the self-energy of that block. It is important to note that $\mathscr{G}_{\mathrm{loc}, i}(\omega)$ contains nonlocal contributions stemming from the off-block-diagonal elements of $H^{\mathrm{TB}}$, that enter $G_{\mathrm{loc}}$ (and hence $G_{\mathrm{loc}, i}$ ) by matrix inversion in Eq. 1.

The impurity Green's function $\mathscr{G}_{i}(\omega)$ allows us to formulate the corresponding Anderson impurity problem, amenable to a numerical solution. Various techniques 
can be used [3,5], yet presently the method of choice is the continuous time quantum Monte Carlo in the hybridization expansion (CT-HYB, see [55] for a review; an implementation for the Kanamori Hamiltonian is discussed in [56]). The main parameters of a CT-HYB calculation are the inverse temperature $\beta\left(\beta=39.6 \mathrm{eV}^{-1}\right.$ corresponds to room temperature), the interaction parameters and the number of Monte Carlo sweeps. After the CT-HYB calculations ( $n$ of them or less, in case some of the atoms are locally equivalent) we access the set of self-energies $\Sigma_{i}(\omega)$ $(0<i \leq n)$. This in turn allows for the construction of a new local Green's function $G_{\text {loc }}$ for the whole heterostructure, as in Eq. 1. Let us stress again that in DMFT the self-energy is frequency-dependent, but does not depend on $\mathbf{k}$, because the impurity model is a local problem. With the new Green's function, we can start another DMFT cycle. This procedure is repeated unit convergence.

The only term that we have not discussed so far is the double-counting correction $H_{\mathrm{DC}}$ (Eq. 1). The underlying idea is to subtract those contributions to $\Sigma_{i}$ that are already accounted for by the GGA. Unfortunately, GGA lacks a diagrammatic description, and such contributions can not be determined rigorously. Several forms of $H_{\text {DC }}$ have been proposed, but no general solution to this conundrum exists, a problem also relevant for DFT $+U$. However, in our calculation with $t_{2 g}$-orbitals only the symmetry is close to cubic where $H_{\mathrm{DC}}$ is equivalent to a trivial shift of the chemical potential $\mu$ [5].

Several remarks concerning the performance should be made. For typical (not too large) supercells, the computationally most time-consuming step is solving the DMFT impurity problems. Here, the crucial parameter is the number of orbitals $(m)$, because for CT-HYB the computational time scales exponentially with $m$. In contrast, the number of sites ( $n$ ) defines the number of impurity problems to solve, and the computational time scales only linearly with $n$. For larger supercells the matrix inversion in Eq. (1) which scales like $(m n)^{3}$ (or best $\sim(m n)^{2.373}$ ) becomes the bottleneck.

\subsection{Comparison with the experiment}

The final step of a DFT+DMFT calculation is postprocessing. CT-QMC calculates Green's functions $G(i \omega)$ on the imaginary frequency (Matsubara) axis and an analytical continuation is required in order to compare with the experimental $A(\omega)$ spectra. Although there exists a one-to-one correspondence between $G(\omega)$ and $G(i \omega)$, a straightforward solution is practically impossible. The root cause of the problem are statistical errors in $G(i \omega)$ that give rise to huge differences in the analytically-continued $G(\omega)$. A standard solution to this problem is the maximum entropy method [57].

After an analytical continuation of the self-energy, the spectral function $A(\mathbf{k}, \omega)$ can be evaluated as

$$
A(k, \omega)=-\frac{1}{\pi} \operatorname{Im} G(k, \omega)=-\frac{1}{\pi} \operatorname{Im}\left[(\omega+\mu) \mathbf{I}_{m} \otimes \mathbf{I}_{n}-H(k)-\Sigma(\omega)\right]^{-1} .
$$




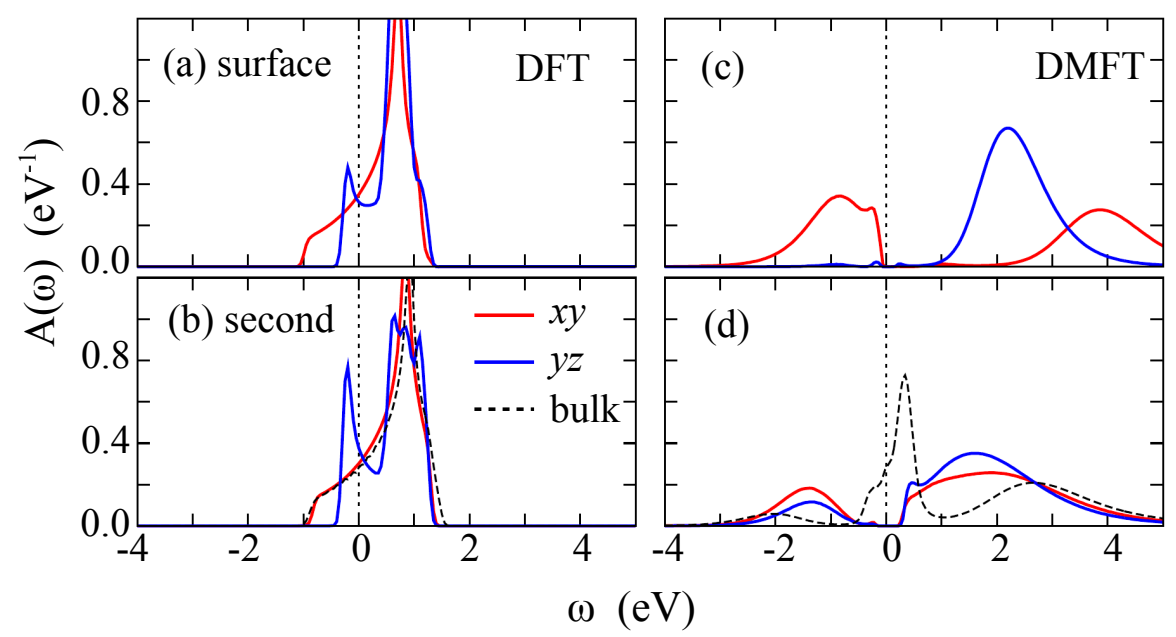

Fig. 3 Layer- and orbital-resolved DOS (left) and the respective DFT+DMFT spectral functions (right) of the $2 \mathrm{SVO} / 4 \mathrm{STO}$ heterostructure, compared to bulk $\mathrm{SrVO}_{3}$ (dashed line) (adapted from [17]).

Orbital occupations can be obtained by integrating the respective diagonal elements of $A(\mathbf{k}, \omega)$ over the Brillouin zone in the negative frequency range up to the Fermi energy.

We are now in the position to compare the DFT+DMFT spectral functions with the DOS and in this way evaluate the effect of electronic correlations. The left panels of Fig. 3 show the layer- and orbital-resolved DOS for the 2 SVO/4 STO heterostructure, with the nonzero DOS at the Fermi level indicating that both layers are metallic. The $x y$ DOS is similar to bulk $\mathrm{SrVO}_{3}$, but for the $y z / x z$ orbitals the confinement in the $z$ direction leads to two peaks in the Fig. $3(\mathrm{a}, \mathrm{b})$ and a narrowing of the DOS. The $y z / x z$ DOS center of mass is shifted upwards to higher energies compare the onsite terms in Table 1. Fig. 3 (right) shows the room-temperature DFT+DMFT spectral functions for $2 \mathrm{SVO} / 4 \mathrm{STO}$. The effect of correlations is dramatic [17]: In the surface layer, they trigger the orbital polarization which renders the $x y$ orbital half-filled. At half-filling, the intraorbital Coulomb repulsion is particularly efficient, and it splits the spectrum into lower and upper Hubbard bands, stabilizing the Mott insulating state. The subsurface layer also becomes insulating, albeit with a weaker orbital polarization and a much narrower gap.

Fig. 4 shows the self-energy $\Sigma(\omega)$ of the 2 SVO / 4 STO heterostructure. Correlations in the $x y$ orbital are particularly strong, as reflected in the sizable frequency dependence of $\operatorname{Re} \Sigma$. At higher frequencies, the DMFT self-energy recovers the static Hartree shift, but it is the low energy part of of $\operatorname{Re} \Sigma$ which deviates strongly for the different orbitals, enhances the DFT crystal field splitting and leads to insulating SVO layers, similar as in [58].

From the metallic-like behavior of $\operatorname{Im} \Sigma$, we can conclude that the $2 \mathrm{SVO} / 4 \mathrm{STO}$ heterostructure is on the verge of a Mott transition. Switching between metallic 


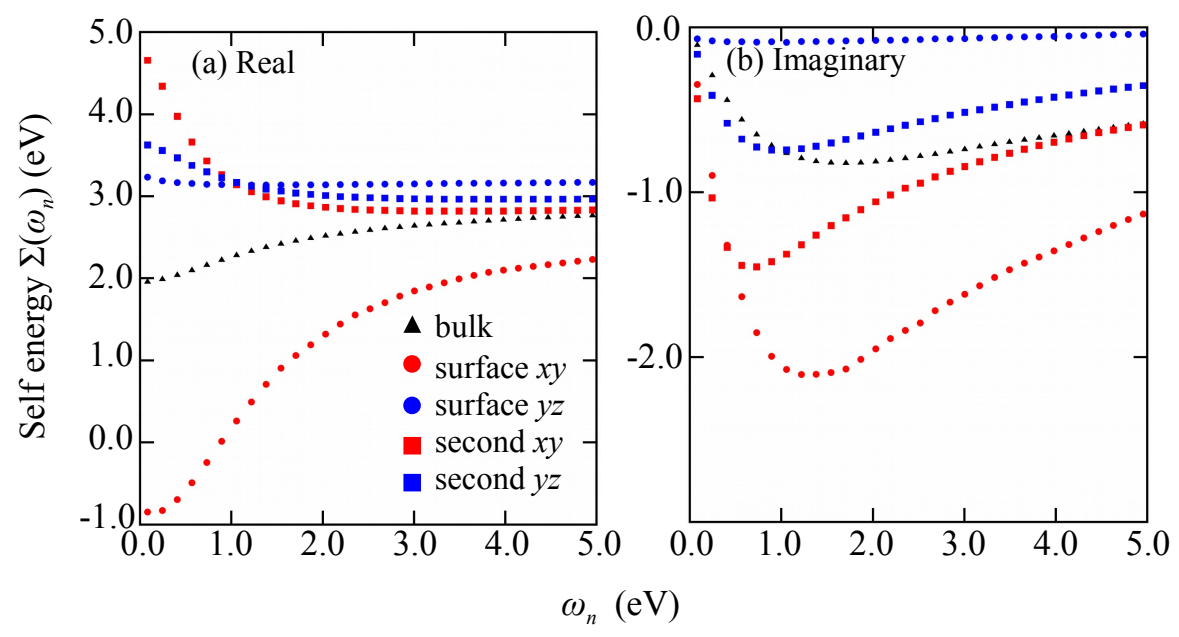

Fig. 4 (a) Real and (b) imaginary part of the layer- and orbital-resolved DMFT self-energy for $2 \mathrm{SVO} / 4 \mathrm{STO}$ as a function of real frequency $\omega$. Results for bulk $\mathrm{SrVO}_{3}$ are shown to illustrate the effect of dimensional reduction.

and insulating regimes can be achieved by strain, doping, applying external electric field, or capping the surface layer. DFT+DMFT is an excellent computational tool to explore these possibilities and evaluate the optimal way to tune the physics of this interesting system [17].

\section{Applications}

After the discovery of the emergent metallicity at the $\mathrm{LaTiO}_{3} / \mathrm{SrTiO}_{3}$ interfaces, one of the major challenges for theory was to explain the mechanism underlying the formation of the metallic state at the interface between a band insulator $\left(\mathrm{SrTiO}_{3}\right)$ and a Mott insulator $\left(\mathrm{LaTiO}_{3}\right)$. This dichotomy motivated Okamoto and Millis to build up the concept of "electronic reconstruction", extending earlier ideas for fullerenes [59] to oxides. The first model calculations [60, 61] along these lines included correlation effects on the Hartree-Fock level. A DMFT model calculation for the $\mathrm{LaTiO}_{3} / \mathrm{SrTiO}_{3}$ heterostructure followed [62], showing a "leakage" of electrons from the Mott- to the band-insulating side. This gives rise to a partially filled $d$-shell at the interface layer which facilitates conductivity. Even though the low-energy model comprised a single orbital only, these results strongly contributed to the understanding of the electronic reconstruction mechanism. Later realistic DFT+DMFT calculations for oxide heterostructures followed, starting with the work of Hansmann et al. for nickelates [63]. In the following four Sections we review DFT+DMFT calculations for oxide heterostructures, which focused hitherto on titanates, nickelates, vanadates, and ruthenates. 


\subsection{Titanates}

Titanates are by far the most studied class of oxide heterostructures. At the same time, they are arguably also the best understood class, and the main ingredients of their emergent behaviors are known from detailed DFT+DMFT studies, pioneered by Lechermann et al. [64, 65, 13, 66]. In particular, this is the case for $\mathrm{LaTiO}_{3} / \mathrm{SrTiO}_{3}$ (LTO/STO) where DFT+DMFT is needed to properly describe the Mott insulator LTO. In the $\mathrm{LaAlO}_{3} / \mathrm{SrTiO}_{3}$ heterostructure on the other hand, which is experimentally most frequently studied, $\mathrm{LaAlO}_{3}$ (LAO) is instead a simple band insulator. The metallic state induced by a slight doping due to oxygen vacancies or electronic reconstruction, is hence only weakly correlated. This situation in LAO/STO can be well described by DFT, except for states localized at the oxygen vacancies.

State-of-the-art DFT+DMFT(CT-HYB) calculations for the LTO/STO heterostructure started with Lechermann et al. [64] who reported the formation of a quasiparticle peak primarily of $d_{x y}$-orbital character, due to the lifted $t_{2 g}$-degeneracy and in line with the experimental observations. A subsequent study focused on the magnetic properties and revealed that ferromagnetism is stabilized by the joint effect of electronic correlations and oxygen vacancies [65]. The role of the latter is a question of fundamental importance, and DFT $+U$ (see e.g. [24]) and DFT+DMFT [13, 66, 67] studies are at the forefront of the research. DFT+DMFT indicates the formation of in-gap states in LAO/STO [65, 66, 67], in good agreement with PES measurements. This is definitely one of the most promising directions of investigation, even though the presence of oxygen vacancies increases the computational effort tremendously. The strongly correlated nature of such defect states gives rise to a strong sensitivity of the electronic properties to small changes in the interaction parameters or local distortions ensuing from the structural relaxation.

Another important aspect are local distortions and rotations of the oxygen octahedra. Their role in stabilizing an insulating state in bulk $d^{1}$ titanates has been advocated by Pavarini et al. [68, 69]. Heterostructuring strongly affects these distortions and rotations because of strain and breaking translational invariance. Dymkowski and Ederer found in DFT+DFMT that compressive strain reduces the $t_{2 g}$-splitting which is intimately related to the collective tilts and rotations, and eventually turns $\mathrm{LaTiO}_{3}$ into a metal [70].

More recently a promising route towards new electronic behaviors, the so-called $\delta$-doping, has been proposed, i.e., adding a single impurity layer into a layered superlattice. To this end, one of the $\mathrm{LaO}$ layers in a large $\mathrm{LaTiO}_{3}$ supercell has been replaced by an $\mathrm{SrO}$ layer and studied using DFT+DMFT [71]. It was found that depending on the distance from the interface, titanate layers exhibit three distinct electronic states [71]. 


\subsection{Nickelates}

Bulk rare-earth nickelates $\mathrm{RNiO}_{3}$ with trivalent $\mathrm{Ni}^{3+}$ exhibit interesting electronic, magnetic and transport properties [72, 73, 74]. One of the most remarkable phenomena is the metal-insulator transition observed in all $\mathrm{RNiO}_{3}$ materials except for $\mathrm{LaNiO}_{3}$ (LNO), which remains metallic down to the lowest temperatures [72]. The nature of this metal-insulator transition has been addressed by experiment $[75,76$, $77,78]$ and theory $[79,80,81,78]$, but the discussion is not yet settled.

In the simplest ionic model, $\mathrm{Ni}^{3+}$ has a $d^{7}$ configuration in LNO. The octahedral crystal field splits the $d$ levels into $t_{2 g}$ and $e_{g}$ manifolds, with the low-lying $t_{2 g}$ states completely filled. Hence all electronic, orbital and spin degrees of freedom pertain to the $e_{g}$ states. Close similarities between nickelates and cuprates were noticed in the early days of high-temperature superconductors. Indeed, the $e_{g}$ shell of $d^{7} \mathrm{Ni}$ with its single electron is seemingly a sibling of $d^{9} \mathrm{Cu}$ having a single hole. Thus, a hypothetical nickelate with half-filled $x^{2}-y^{2}$ and empty $3 z^{2}-r^{2}$ orbitals would have a cuprate-like Fermi surface and eventually become superconducting upon doping. But bulk $\mathrm{Ni}^{3+}$ systems do not exhibit such an orbital polarization [82].

Chaloupka and Khaliulin [83] suggested to resort instead to nickelate heterostructures, with the idea in mind that the $z$ axis confinement leads to a reduced bandwidth of the $3 z^{2}-r^{2}$ orbital which hence gets Mott insulating, leaving behind a $x^{2}-y^{2}$ Fermi surface. In realistic DFT+DMFT calculations, Hansmann et al. [63] found that it is instead the correlation enhanced crystal field splitting between $x^{2}-y^{2}$ and $3 z^{2}-r^{2}$ orbitals which pushes the $3 z^{2}-r^{2}$ orbital higher in energy and gives rise to singlesheet Fermi surface of $x^{2}-y^{2}$ character. However, strain or a $\mathrm{PrScO}_{3}$ substrates is needed to achieve this, or unrealistically large $U$ values [63]. This can also trigger a metal-insulator transition which is very different for the $x^{2}-y^{2}$ and $3 z^{2}-r^{2}$ orbital [84]: While $\operatorname{Im} \Sigma_{x^{2}-y^{2}}(\omega)$ diverges for $\omega \rightarrow 0$ as in the single-band Hubbard model, $\operatorname{Im} \Sigma_{3 z^{2}-r^{2}}(\omega)$ retains a seemingly metallic-like behavior. The insulating state is instead induced by the change in $\operatorname{Re} \Sigma_{3 z^{2}-r^{2}}(\omega)$ relative to $\operatorname{Re} \Sigma_{x^{2}-y^{2}}(\omega)$ at $\omega=0$, i.e., by the aforementioned enhancement of the crystal field splitting.

Later, Han el al. [85] challenged this picture and vouched for an explicit inclusion of the oxygen ligand orbitals into the calculation. Their study found electronic correlations to decrease the orbital polarization disregarding the initial crystal-field splitting. The main difference between the two DMFT studies is the choice of the basis: Hansmann et al. employed a $d$-only calculation including the $\mathrm{Ni} e_{g}$ states and which only requires a minimal number of interaction parameters. In contrast, Han et al. [85] employed a $d$ - $p$ basis, where the O $2 p$ orbitals are included explicitly. The choice of the $d$ - $p$ model is motivated by the high oxidation state of $\mathrm{Ni}^{3+}$, which can be unstable towards the formation of a ligand hole (L) and the transfer of an electron to the Ni $d$ shell. In the $d$-p calculation, which allows for such charge-transfer processes, the strong hybridization between $\mathrm{Ni} d$ and $\mathrm{O} p$ states and the Hund's exchange in the $d$-shell reduce the orbital polarization [85]. In principle, including additionally the $\mathrm{O} p$ states should be better as it allows for charge transfer processes. However, such calculations are very sensitive to the double counting or $d$ - $p$ interaction and a commonly accepted scheme still needs to be established. 
An extensive comparative DMFT study of $d$ and $d$-p models allowed Parragh et al. [86] to pinpoint the difference between the two models [86], see Fig. 5. Parragh $e t$ al. found that the deviations are ruled by the filling of the correlated $d$-shell. In the $d$-only model the filling is $d^{7}$ or one electron per site in the two $e_{g}$ orbitals so that shifting the second $3 z^{2}-r^{2}$ orbital upwards and reducing its occupation is energetically favorable (Fig. 5 a). For the $d$ - $p$ model instead an essentially $d^{8}$ (ligand hole) configuration brings the Hund's exchange into the game. This favors the two $e_{g}$ electrons to form a spin $S=1$, equally occupying the two different $3 z^{2}-r^{2}$ and $x^{2}-y^{2}$ orbitals (Fig. 5 b). This actually leads to a downward instead of an upward shift of the $3 z^{2}-r^{2}$ orbital [86]. This has dramatic consequences for the topology of the Fermi surfaces: while the $d$-only model features a single-sheet cuprate-like Fermi surface (Fig. 5 c), a second sheet always appears in $d$ - $p$ model calculations (Fig. 5 d). Later Peil et al. applied DFT+DMFT to study strain effects in nickelate superlattices [87]. As in Ref. [63] they found that strain can induce a sizable orbital polarization. However they point out that the Hund's exchange can reduce the orbital polarization for both the $d^{8} L$ and $d^{7}$ configuration. The latter $d^{7}$ result revises common knowledge and is an indirect consequence of the intersite hopping. Noteworthy, their DMFT orbital polarization is smaller than in DFT.

Which of the two calculations, $d$-only or $d$ - $p$, provides a more appropriate framework for the fermiology and spin-state of nickelate heterostructures? From the physical perspective, the $d$-p model is clearly superior to the $d$-only model: Owing to the larger energy window used for Wannier projections, the resulting Wannier functions are more localized and atomic-like. In addition, the occupation of the $d$-shell in $d$-p models is flexible, and hence such models can capture charge transfer. However, the price to pay is the drastic increase in the number of free parameters, including the on-site interactions on the $\mathrm{O} 2 p$ orbitals as well as $d$ - $p$ interaction whose experimental estimation is very challenging. Or, alternatively, a still tentative double counting or an $a d$ hoc adjustment of the $\mathrm{O} p$ level to experiment is necessary.

On the experimental forefront, the situation remains controversial. In LAO/LNO heterostructures, orbital polarizations (but only moderate ones) have been found. For $4 \mathrm{LNO} / 4 \mathrm{LAO}$ heterostructures, soft x-ray reflectivity measurements reveal a small orbital polarization of 7(4) \% for the outer (inner) LNO monolayers [88]. On the other hand, by choosing a different non-correlated spacer, such as e.g. $\mathrm{GdScO}_{3}$, tan orbital polarization as high as $25 \%$ can be achieved [89]. Recently grown three-component LTO/LNO/LAO heterostructures show even larger polarizations [90]. The origin of sizable orbital polarization in these heterostructures has been addressed in a very recent DFT+DMFT study [91]. In the layered bulk nickelate $\mathrm{Eu}_{2 x} \mathrm{Sr}_{x} \mathrm{NiO}_{4}$, Uchida et al. [92] found a single cuprate-like Fermi surface in ARPES which even evidences a pseudogap. Experimentally the smoking gun would be to determine the local magnetic moment which should be $\sim 2$ and $\sim 1 \mu_{\mathrm{B}}$ for the $d^{8} L$ and $d^{7}$ configuration, respectively. 

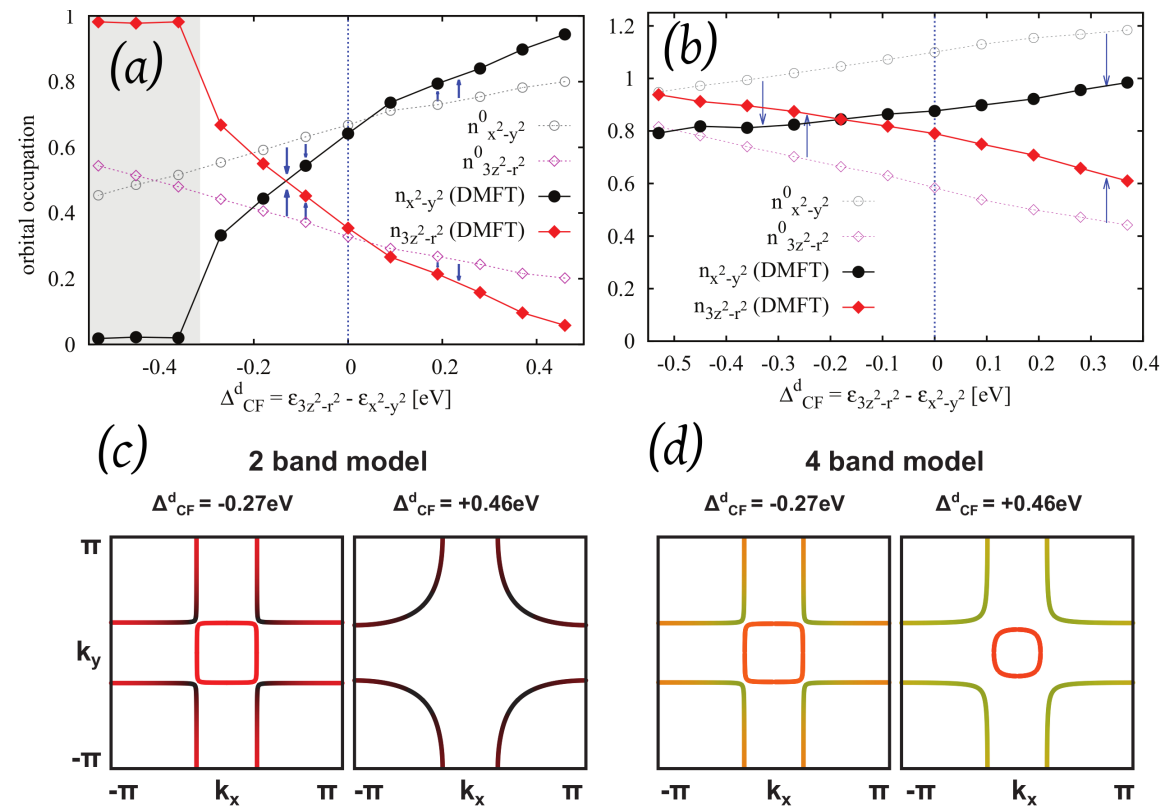

(d)

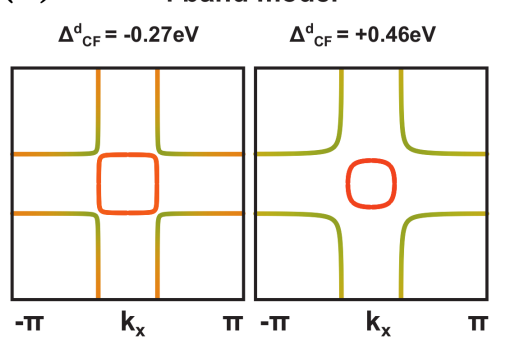

Fig. 5 (a) Orbital occupations vs. crystal field splitting for a $d$-only ( 2 band) model of a LNO monolayer without interaction (open symbols) and within DMFT (filled symbols; $U=5.5 \mathrm{eV}$, $J=0.75 \mathrm{eV}, T=1165 \mathrm{~K}, n_{e}=1$ electron/site). The shaded region denotes the Mott-Hubbard insulating phase. (b) Same for a $d-p$ (4 band) model $\left(U=10 \mathrm{eV}, n_{e}=5\right.$ ). (c-d) Shape and orbital character of the Fermi surface as calculated by DMFT for (c) the $d$-only and (d) the $d$ - $p$ model. The color coding is as follows: black for $x^{2}-y^{2}$, red for $3 z^{2}-r^{2}$, yellow for $\mathrm{O} p$. For the $d$-only model electronic correaltions enhance the orbital disproportionation (a) while for the $d$ - $p$ model it is reduced, preventing a single-sheet Fermi surface (d) as in (c). (adapted from [86]).

\subsection{Vanadates}

In this section we review DFT+ $U$ and DFT+DMFT calculations of $\mathrm{LaVO}_{3}(\mathrm{LVO})$ heterostructures. Please note that results for $\mathrm{SrVO}_{3} / \mathrm{SrTiO}_{3}$ which might serve as a Mott transistor [17] have already been discussed in Section 2.2; also the charge transfer in $\mathrm{SrVO}_{3} / \mathrm{SrMnO}_{3}$ has been analyzed [93]. Bulk LVO has been analyzed within DFT $+U$ by Fang and Nagaosa [94] and within DFT+DMFT by De Raychaudhury, et al. [95]. The latter calculation revealed the existence of strong orbital fluctuations above the Néel temperature $T_{N}$, which coincides with the structural transition temperature. These quantum effects reduce quite rapidly the orbital polarization, in contrast to $\mathrm{YVO}_{3}$, for instance, where a pronounced orbital polarization persists up to temperatures of the order of $1000 \mathrm{~K}$, i.e. well above $T_{N}$. The magnetism in LVO is of $C$-type, i.e. ferromagnetically stacked planes with antiferromagnetic order within each plane $(q=\pi \pi 0)$. This ordering is accompanied by an orbital pattern of an intermediate kind between $G$ - and $C$-type, due to the competition between the Jahn-Teller of the oxygen octahedra and the $\mathrm{GdFeO}_{3}$-type distortion [95]. Note that 


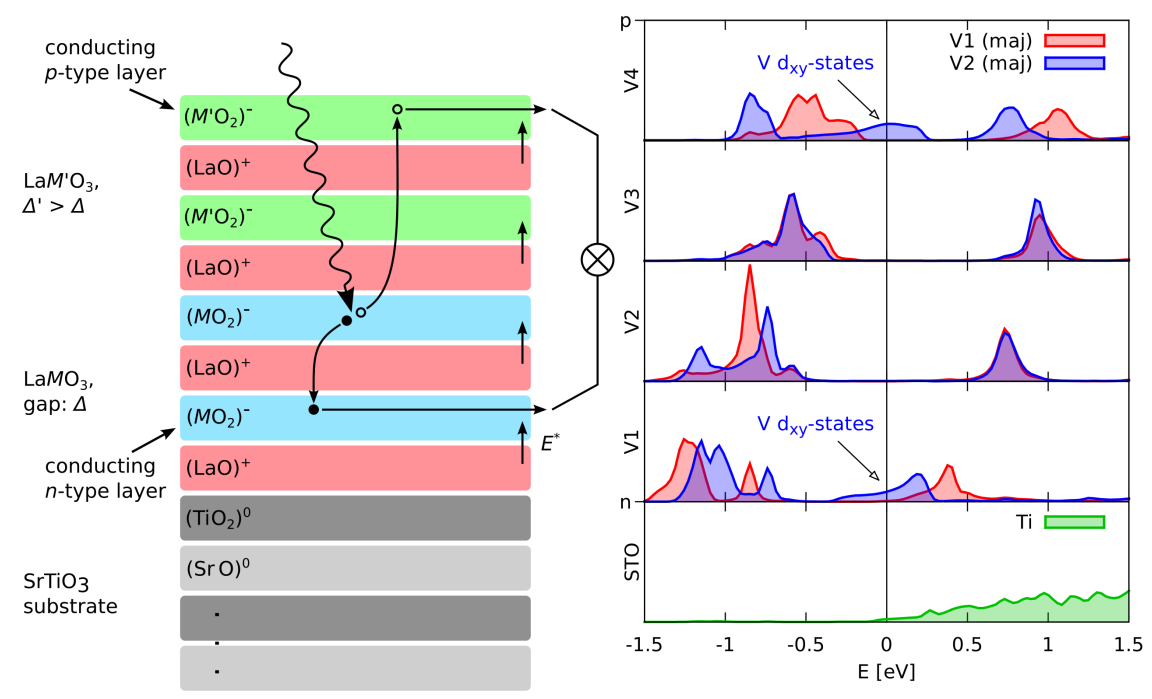

Fig. 6 Left: Proposal of solar cell made out of an oxide heterostructure with a polar discontinuity. The emerging electric field $E$ separates electrons and holes that are generated through the photoelectric effect in the $\mathrm{LaMO}_{3}$. Using different layers with e.g. $\mathrm{M}=\mathrm{V}, \mathrm{M}^{\prime}=\mathrm{Fe}$ allows for a gap grading. Right: DFT $+U$ calculation for four layer of LVO on top of a STO substrate (only the topmost STO layer is shown). The layer-resolved DOS shows the polar field as a shift of the upper and lower Hubbard bands and an electronic reconstruction giving rise to metallic interface and surface layers (adapted from [97]).

in SVO/LVO heterostructures also a ferromagnetic state is possible. The structural transition at $140 \mathrm{~K}$ is from a high-temperature orthorhombic to a low-temperature monoclinic phase.

Hotta et al. [96] were able to grow LVO epitaxially on STO and found a metallic $n$-type interface for thick enough LVO films, which they interpreted to originate from the polar discontinuity. Motivated by these experiment and also intrigued by the advantageous size of the gap of LVO $(\sim 1 \mathrm{eV})$ for photovoltaic applications, Assmann et al. studied LVO thin films with DFT $+U$ [97] and DFT+DMFT [98].

Fig. 6 (left panel) shows the scheme proposed in Ref. [97] for a solar cell with transition-metal oxide perovskites as absorbing materials. The main idea of the $\mathrm{LaVO}_{3}$-based solar cells is that electrons and holes produced by the incoming photon can be efficiently separated and harvested due to the polarity of the heterostructure with its intrinsic potential gradient (indicated as an electric field $E$ in the left panel of Fig. 6). The DFT $+U$ results for 4 LVO layers on top of a STO substrate are shown in Fig. 6 (right panel). The position of the lower and upper LVO Hubbard band shifts from layer to layer, indicating the polar electric field which can separate photoelectrically induced electrons and holes. Above a critical thickness of four layers, the valence and conduction $d$-bands of vanadium touch the Fermi level, which leads to an electronic reconstruction and a metallic interface and surface layer. The latter might need protection from a capping layer to suppress disorder effects and 
achieve a sufficient electron mobility. In a solar cell, these metallic layers may be exploited to transport the electron and holes to the power consumer.

The optical absorption which indicates the efficiency of the photoelectric field in the LVO-STO heterostructure was calculated in Ref. [97]. The results compare quite favorably with the most modern thin-film solar cell materials. Particular appealing is that oxide heterostructures can overcome the infamous SchockleyQueisser limit of 38\% efficiencies in two ways: (i) As indicated in Fig. 6 (left panel) different transition metals $\mathrm{M}$ and $\mathrm{M}^{\prime}$ may lead to different gaps $\left(\mathrm{LaFeO}_{3}\right.$ and $\mathrm{LaVO}_{3}$ for instance were considered in Ref. [97]). Such a "band-grading" is very flexible in oxide heterostructures and can overcome loosing the excess photon energy beyond the band gap to photons (heating up the solar cell instead of gaining electric energy). (ii) A genuine correlation effect, impact ionization, creates additional electron-hole pairs on the $10 \mathrm{fs}$ time scale [99] if the photon energy is larger than twice the band gap.

Recently, Wang, et al., [100] reported the realization of an actual solar cell, where $\mathrm{LaVO}_{3}$ serves as the light absorber. Further work on the quality of the heterostructure samples is needed to enhance the efficiency of the solar cell which still suffers from a poor mean free path is only comparable to the dawn of Si solar cells. Also for the other proposed material, $\mathrm{LaFeO}_{3}$ on $\mathrm{SrTiO}_{3}$, Nakamura et al. [101] reported its photovoltaic applicability.

While it is unclear at present whether oxide heterostructures may become a viable alternative to Si solar cells, the proposal and experimental confirmation already served another purpose: It confirms the existence of a polar field. The latter has been controversially debated recently, in particular in view of the fact that oxygen vacancies counteract the polar field and also may induce a metallic interface layer, e.g. in LAO/STO. Demonstrating a actually working solar cell proves at a polar field survives in various oxide heterostructures.

\subsection{Ruthenates}

Bulk $\mathrm{SrRuO}_{3}$ (SRO) is a rare example of a ferromagnetic conducting oxide [102], and there is a long standing experimental experience in growing films of this perovskite: thin single-domain films of high quality can be grown with a pure $\mathrm{SrO}$ termination, which is assisted by the volatility of $\mathrm{RuO}_{3}$ and $\mathrm{RuO}_{4}$ oxides [103]. A typical substrate is STO with its cubic lattice constant $a=3.905 \AA$ giving rise to to a moderate compressive strain $(\sim 0.45 \%)$ in SRO [102].

Resistivity measurements on ultrathin SRO layers show a pronounced dependence on the layer thickness: while the bulk behavior is recovered in thick slabs of $\gtrsim 15$ SRO layers, fewer layers show a reduced Curie temperature and a strongly enhanced resistivity [104]. Drastic changes occur upon reaching the critical thickness of four monolayers, at which SRO turns into a non-magnetic insulator [105]. Experimental attempts to stabilize ferromagnetism in single (or a few) SRO layers by capping or applying compressive/tensile strain remain unsuccessful so far. 
Since such ultrathin ferromagnetic films are important for prospective (e.g. spintronics) applications, understanding the nature of the metal-insulator transition and the absence of ferromagnetism is mandatory. Quite remarkably, neither the electronic ground state, nor an antiferromagnetism or otherwise non-ferromagnetic phase are captured by DFT: it yields a ferromagnetic state for all slabs thicker than one SRO monolayer and fails to reproduce the insulating state [106]. Similarly, $\mathrm{DFT}+U$ yields a metal even for sizable $U$ values [106]. Later studies showed that an antiferromagnetic insulator can be stabilized in DFT $+U$ for the spurious $\mathrm{RuO}_{2}$ termination [107], which is however in contrast to the experiment [108]. Moreover, $\mathrm{DFT}+U$ yields antiferromagnetism for layers of up to eight SRO monolayers, i.e. substantially overestimates the experimental critical thickness.

These apparent shortcomings of DFT and DFT $+U$ call for a more realistic treatment of electronic correlations within DFT+DMFT. The latter accounts for the behavior of bulk $\mathrm{SrRuO}_{3}[109,110]$, and although DMFT is a cruder approximation in $2 \mathrm{D}$ than in 3D, we can still expect it to capture the physics of thin SRO layers including its magnetism. The influence of the SRO layer thickness onto the electronic and magnetic ground states was studied very recently in Ref. [111]. All DFT calculations were done using a $\sqrt{2} \times \sqrt{2} \times 6$ supercell, which allows us to study SRO/STO compositions ranging from a SRO monolayer (1:5) to a four-layer slab (4:2) and is compatible with both ferromagnetic and antiferromagnetic in-plane order. The out-of-plane unit cell parameter as well as the internal atomic coordinates were optimized using DFT, while keeping the in-plane lattice constant equal to that of the STO substrate. The wien2wannier[53]-derived Hamiltonian was supplemented with a Coulomb repulsion $U=3.5 \mathrm{eV}$ and Hund's exchange $J=0.3 \mathrm{eV}$ as derived by the constrained random phase approximation (cRPA) approach. The resulting interacting Hamiltonians were treated in DMFT and solved using a CT-HYB solver.

For SRO monolayers and bilayers, DFT+DMFT readily yields an insulating antiferromagnetic state at room temperature (Fig. 7 d). The microscopic origin of this state lies in the sizable orbital polarization: the $x y$ orbital is occupied by two electrons, while the $x z$ and $y z$ orbitals are half-filled, see Fig. 7 a-c. In accord with the Hund's rule, the spins of the latter two orbitals align parallel, giving rise to a localized magnetic moment of $\sim 2 \mu_{\mathrm{B}}$. While in a ferromagnetic configuration the $x z$ and $y z$ electrons are essentially immobile (Fig. 7 b), in the antiferromagnetic phase they can hop between the neighboring Ru sites gaining kinetic energy through superexchange processes (Fig. 7 c). Hence DMFT does not only agree with experiment, but also explains the metal-insulator and ferromagnetic-antiferromagnetic transition upon reducing the thickness of SRO films.

Unlike DFT and DFT $+U$, DMFT allows us to study the temperature evolution of electronic and magnetic states. In this way, it was shown that SRO monolayers retain a sizable orbital polarization even in the paramagnetic state $(\sim 1000 \mathrm{~K})$. Indeed, a strong tendency towards orbital polarization is visible already at the DFT level, marked by the difference between the on-site energies of the $x y$ orbital and the degenerate $x z$ and $y z$ orbitals. This situation is typical for thin films confined along the $z$ direction but apparently different from bulk SRO, where the three $t_{2 g}$ orbitals are degenerate (Fig. 7 a). This effect is somewhat countered by the elongation of the 

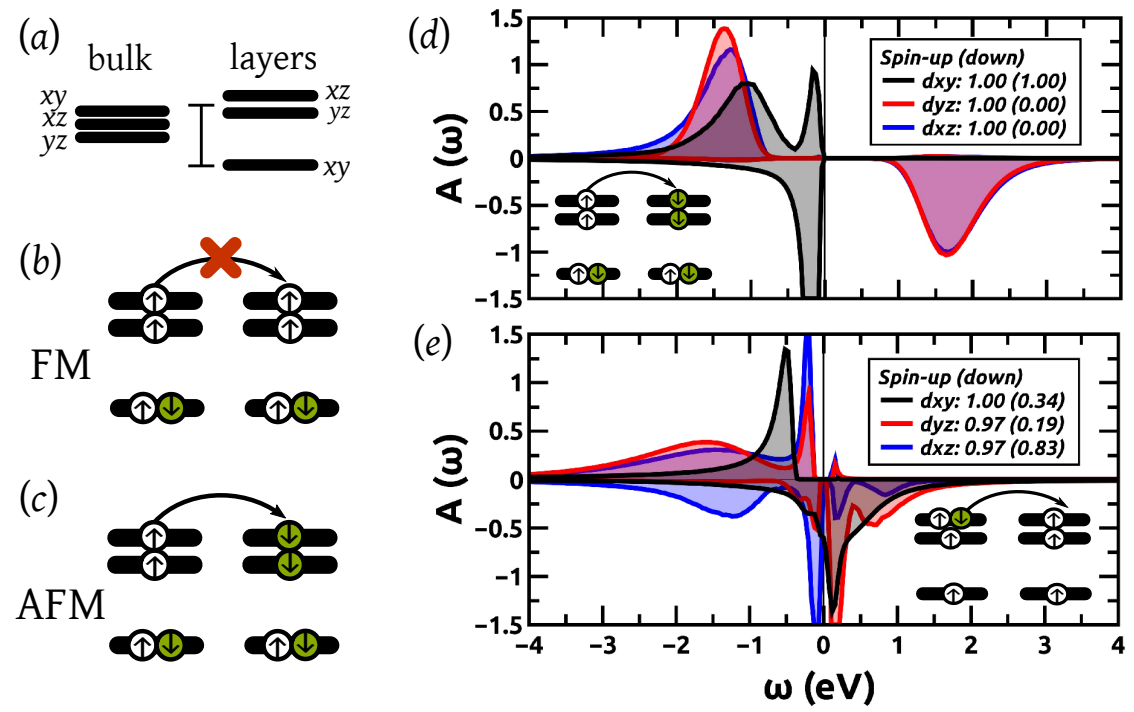

Fig. 7 Ramifications of the dimensional reduction in $\mathrm{SrRuO}_{3}$ thin films. (a) The degeneracy of the three $t_{2 g}$ in the bulk is lifted for ultrathin layers. (b) For a ferromagnetic configuration there is hence no hopping, while for (c) an antiferromagnetic configuration virtual (superexchange) hoppings within the $t_{2 g}$ manifold are allowed. This explains why antiferromagnetism is energetically favorable in SRO thin films. (d-e) Orbital-resolved DFT+DMFT spectral function $A$ as a function of real frequency $\omega$ for (d) one SRO monolayer on a STO substrate revealing an antiferromagnetic insulating state, and (e) an electron-doping with 4.3electrons/Ru turning the SRO film into a half-metallic ferromagnet (adapted from [111]).

topmost $\mathrm{RuO}_{6}$ octahedra, which in the crystal-field picture lowers the energy of $x z$ and $y z$ orbitals. However, this effect is weak and the $x y$ orbital always lies lower in energy [111].

Can DFT+DMFT calculations give a clue to the quest of growing ultrathin ferromagnetic SRO films? A standard way to manipulate physical properties of thin films is the strain induced by the lattice mismatch between the film and the substrate. Unfortunately, DMFT calculations reveal that for SRO neither compressive nor tensile strain stabilizes an FM state, at least for realistic values of the on-site Coulomb repulsion [111]. Another possibility to bring on-site energies closer together is capping SRO with STO layers, which should to a certain extent restore the bulk behavior. Although the level splitting is indeed reduced in DFT, DMFT still yields an antiferromagnetic state [111], albeit with now somewhat more balanced orbital occupations. Thus, the antiferromagnetism is a robust feature of SRO layers: neither strain nor capping can stabilize the desired ferromagnetic state. This explains why experimental attempts to fabricate ferromagnetic SRO layers were hitherto unsuccessful.

There is however an alternative route to restore ferromagnetism: doping. To verify this scenario, additional DFT calculations were performed within the virtual crystal approximation, mimicking the $\mathrm{Ru} d$-level occupation of 3.7 electrons. For 
this filling, DMFT indeed yields a half-metallic ferromagnet (Fig. 7 e). Quite remarkably, for both, electron and hole doping, the magnetic moments and the magnetic ordering temperatures is higher than in bulk SRO [111].

The good agreement between DFT+DMFT and experiment for the undoped phase leaves little doubt that DMFT provides a realistic description of thin SRO layers. Still, there are many open issues that deserve further studies with computational methods, including DMFT. The first and arguably most relevant is the effect of oxygen vacancies. Real materials are never perfect, and particularly thin films are often subject to surface contaminations, growth defects and vacancies. Oxygen vacancies represent a severe experimental challenge: their concentration is difficult to estimate, while the presence of a single vacancy can dramatically alter the properties of the surrounding atoms. Structural relaxations invoked by vacancies can be studied by DFT, yet the alteration of the Ru $4 d$-level filling and its ramifications pertain to correlated electrons and require an appropriate many-body treatment. DFT+DMFT is a state-of-the-art tool for such problems.

\section{Conclusion and outlook}

DMFT is state-of-the-art for dealing with electronic correlations which are of prime importance for many oxide heterostructures; and its merger with DFT [4, 5] allows us to do realistic materials calculations. This way quantum correlations in time are included, corresponding to a local self energy which might differ from site to site. Physically, DFT+DMFT reliably describes among others, quasiparticlerenormalizations, metal-insulator transitions, correlation enhanced crystal field splittings, charge transfers, and magnetism. On the other hand, non-local correlations are neglected. These are important in the vicinity of phase transitions or to describe the physics of excitons and weak localization corrections to the conductivity. In the future, cluster [27] or diagrammatic extensions [29, 30] of DMFT, which are computationally more demanding, and hence hitherto focused on model Hamiltonians, will also be applied to oxide heterostructures.

In the first part, Section 2, we illustrated all steps of a DFT+DMFT calculation for a showcase heterostructure: two layers of SVO on an STO substrate. This starts with a DFT calculation, here using Wien2K [44]. Subsequently a projection onto Wannier orbitals and a corresponding low energy Hamiltonian is performed with the help of wien 2 wannier [53] (integrated in the most recent Wien2K version) and Wannier90 [52]. This may result in a $d$-only Hamiltonian but also additional e.g. oxygen $p$ orbitals can be included. The Wannier projection also defines what is (site-)local in DMFT. For the subsequent DMFT calculation we used w2dynamics [56], ${ }^{2}$ which solves the DMFT impurity problem by continuous-time quantum Monte Carlo simulations [55] in the hybridization expansion. This yields the DFT+DMFT self energy and spectrum. For calculating (optical) conductivities and thermal responses,

2 w2dynamics will be made available by Gnu Public License next year, betatest versions are available upon email request. 
postprocessing with woptics[112] is needed; a DFT+DMFT charge self consistency is possible[113].

In the second part, Section 3, we reviewed previous DFT+DMFT calculations for oxide heterostructures. Not only experimentally but also regarding DMFT calculations arguably most well studied are titanates, in particular, LAO/STO and LTO/STO. Here the former is only weakly correlated whereas electronic correlations are strong for the latter as LTO is a Mott insulator. DMFT describes the formation of a two dimensional electron gas at the interface due to an electronic reconstruction, but also the localization of electrons at oxygen vacancies if these are included in the supercell.

Nickelate-based heterostructures may be used to turn the nickel Fermi surface into a cuprate-like one [84]. This might however be prevented by charge transfer processes: i.e. oxygen ligand holes may lead to a $d^{8} L$ instead of a $d^{7}$ configuration [85]. The physics is completely different [86], spin- 1 instead of spin-1/2, and the theoretical uncertainty regarding the oxygen- $p$ position is too large to reliably predict which scenario prevails. The smoking gun experiment in this respect is measuring the short-time magnetic moment (spin) e.g. by neutron scattering or x-ray absorption spectroscopy.

Turning to vanadates, LVO/STO is a promising candidate for efficient solar cells because of the size of the LVO Mott-Hubbard gap and the polar electric field of the heterostructure which has been suggested [97] and experimentally verified [100] to separate photovoltaically generated electron-hole pairs. SVO on the other hand is a strongly correlated metal in the bulk. With a dimensional reduction it turns insulating below a critical thickness of $\sim 3$ layers [36, 8]. DFT+DMFT [17] could trace back the microscopic origin of this metal-insulator transition to the correlation enhanced crystal field splitting and showed that this transition can also be triggered by a small gate voltage. This makes SVO/STO an ideal candidate for a Mott transistor.

Ruthenates such as SRO/STO are promising for heterostructures that are both metallic and ferromagnetic. DFT+DMFT [111] could explain why the experimental efforts to get ultrathin ferromagnetic SRO/STO failed so-far, despite predictions of the contrary: While bulk SRO is a metallic ferromagnet in DMFT, the correlation enhanced crystal field splitting makes a competing antiferromagnetic phase favorable. This antiferromagnetic phase is quite stable against strain, capping layers etc. The most promising route to metallic ferromagnetism is electron doping.

We have only seen the beginning of DFT+DMFT calculations for oxide heterostructures. In the future, these calculation will help us to better understand experiment and to predict novel physics, which already led to some successes in the past. While the numerical effort is larger than DFT, it is still much more efficient and cheaper than experiment to scan the myriad of possible combinations of hetrerostructure slabs by DFT+DMFT. in experiment and necessitate a refinement of the DFT+DMFT calculation. In the past the theoretical and experimental focus has been on heterostructures with layers perpendicular to the (001) direction. Now both experiment and theory turn to other geometries: confinement in the (110) direction shows a much more complicated quantization and flat bands [10]; bilayers in a (111) stacking on the other hand are interesting regarding prospective topological 
states [114]. With the close cooperation between experiment on the one side and DFT as well as DMFT theory on the other side, a bright future in the research area of oxide heterostructures lies ahead.

We thank our colleagues and coauthors E. Assmann, S. Bhandary, P. Blaha, P. Hansmann, R. Laskowski, G. Li, S. Okamoto, N. Parragh, L. Si, J. Tomczak, A. Toschi, and M. Wallerberger for useful discussions and joint efforts. This work was supported financially by European Research Council under the European Union's Seventh Framework Program (FP/2007-2013)/ERC grant agreement n. 306447, the Austrian Science Fund (FWF) through SFB ViCoM F41 and project I-610, as well as research group FOR1346 of the Deutsche Forschungsgemeinschaft (DFG). Calculations reported were performed on the Vienna Scientific Cluster (VSC).

\section{References}

[1] W. Metzner and D. Vollhardt, Correlated lattice fermions in $\mathrm{d}=\infty$ dimensions, Phys. Rev. Lett. 62, 324 (1989).

[2] A. Georges and G. Kotliar, Hubbard model in infinite dimensions, Phys. Rev. B 45, 6479 (1992).

[3] A. Georges, G. Kotliar, W. Krauth and M. J. Rozenberg, Dynamical meanfield theory of strongly correlated fermion systems and the limit of infinite dimensions, Rev. Mod. Phys. 68, 13 (1996), arXiv:cond-mat/9510091.

[4] G. Kotliar, S. Y. Savrasov, K. Haule, V. S. Oudovenko, O. Parcollet and C. A. Marianetti, Electronic structure calculations with dynamical mean-field theory, Rev. Mod. Phys. 78, 865 (2006), arXiv:cond-mat/0511085.

[5] K. Held, Electronic structure calculations using dynamical mean field theory, Adv. Phys. 56, 829 (2007), arXiv:cond-mat/0511293.

[6] A. Ohtomo and H. Y. Hwang, A high-mobility electron gas at the $\mathrm{LaAlO}_{3} / \mathrm{SrTiO}_{3}$ heterointerface, Nature (London) 427, 423 (2004).

[7] Z. Zhong, Q. Zhang and K. Held, Quantum confinement in perovskite oxide heterostructures: Tight binding instead of a nearly free electron picture, Phys. Rev. B 88, 125401 (2013), arXiv:1308.2615.

[8] K. Yoshimatsu, K. Horiba, H. Kumigashir, T. Yoshida, A. Fujimori and M. Oshima, Metallic quantum well states in artificial structures of strongly correlated oxide, Science 333, 319 (2011).

[9] A. F. Santander-Syro, O. Copie, T. Kondo, F. Fortuna, S. Pailhés, R. Weht, X. G. Qiu, F. Bertran, A. Nicolaou, A. Taleb-Ibrahimi, P. L. Févre, G. Herranz, M. Bibes, N. Reyren, Y. Apertet, P. Lecoeur, A. Barthélémy and M. J. Rozenberg, Two-dimensional electron gas with universal subbands at the surface of $\mathrm{SrTiO}_{3}$, Nature (London) 469, 189 (2011), arXiv:1009.3412.

[10] Z. Wang, Z. Zhong, X. Hao, S. Gerhold, B. Stoger, M. Schmid, J. SanchezBarriga, A. Varykhalov, C. Franchini, K. Held and U. Diebold, Anisotropic two-dimensional electron gas at $\mathrm{SrTiO}_{3}(110)$ protected by its native overlayer, Proc. Nat. Acad. Sci. 333, 3933 (2014), arXiv:1309.7042. 
[11] G. Berner, M. Sing, H. Fujiwara, A. Yasui, Y. Saitoh, A. Yamasaki, Y. Nishitani, A. Sekiyama, N. Pavlenko, T. Kopp, C. Richter, J. Mannhart, S. Suga and $\mathrm{R}$. Claessen, Direct $k$-space mapping of the electronic structure in an oxideoxide interface, Phys. Rev. Lett. 110, 247601 (2013), arXiv:1301.2824.

[12] G. Berner, A. Müller, F. Pfaff, J. Walde, C. Richter, J. Mannhart, S. Thiess, A. Gloskovskii, W. Drube, M. Sing and R. Claessen, Band alignment in $\mathrm{LaAlO}_{3} / \mathrm{SrTiO}_{3}$ oxide heterostructures inferred from hard x-ray photoelectron spectroscopy, Phys. Rev. B 88, 115111 (2013).

[13] M. Behrmann and F. Lechermann, Interface exchange processes in $\mathrm{LaAlO}_{3} / \mathrm{SrTiO}_{3}$ induced by oxygen vacancies, Phys. Rev. B 92, 125148 (2015), arXiv:1506.07066.

[14] H. O. Jeschke, J. Shen and R. Valentí, Localized versus itinerant states created by multiple oxygen vacancies in $\mathrm{SrTiO}_{3}$, New J. Phys. 17, 023034 (2015), arXiv: 1407.7060.

[15] M. Imada, A. Fujimori and Y. Tokura, Metal-insulator transitions, Rev. Mod. Phys. 70, 1039 (1998).

[16] A. Liebsch, Surface versus bulk Coulomb correlations in photoemission spectra of $\mathrm{SrVO}_{3}$ and $\mathrm{CaVO}_{3}$, Phys. Rev. Lett. 90, 096401 (2003), arXiv:condmat/0211257.

[17] Z. Zhong, M. Wallerberger, J. M. Tomczak, C. Taranto, N. Parragh, A. Toschi, G. Sangiovanni and K. Held, Electronics with correlated oxides: $\mathrm{SrVO}_{3} / \mathrm{SrTiO}_{3}$ as a Mott transistor, Phys. Rev. Lett. 114, 246401 (2015), arXiv:1312.5989.

[18] G. Lantz, M. Hajlaoui, E. Papalazarou, V. L. R. Jacques, A. Mazzotti, M. Marsi, S. Lupi, M. Amati, L. Gregoratti, L. Si, Z. Zhong and K. Held, Surface effects on the Mott-Hubbard transition in archetypal $\mathrm{V}_{2} \mathrm{O}_{3}$, Phys. Rev. Lett. 115, 236802 (2015), arXiv:1507.02116.

[19] Z. P. Yin, K. Haule and G. Kotliar, Kinetic frustration and the nature of the magnetic and paramagnetic states in iron pnictides and iron chalcogenides, Nat. Phys. 10, 932 (2011), arXiv:1104.3454.

[20] A. Georges, L. de' Medici and J. Mravlje, Strong electronic correlations from Hund's coupling, Ann. Rev. Condens. Matter Phys. 4, 137 (2013), arXiv:1207.3033.

[21] V. I. Anisimov, J. Zaanen and O. K. Andersen, Band theory and Mott insulators: Hubbard $U$ instead of Stoner I, Phys. Rev. B 44, 943 (1991).

[22] G. Sangiovanni, A. Toschi, E. Koch, K. Held, M. Capone, C. Castellani, O. Gunnarsson, S.-K. Mo, J. W. Allen, H.-D. Kim, A. Sekiyama, A. Yamasaki, S. Suga and P. Metcalf, Static versus dynamical mean-field theory of Mott antiferromagnets, Phys. Rev. B 73, 205121 (2006), arXiv:condmat/0511442 .

[23] D. D. Cuong, B. Lee, K. M. Choi, H.-S. Ahn, S. Han and J. Lee, Oxygen vacancy clustering and electron localization in oxygen-deficient $\mathrm{SrTiO}_{3}$ : LDA+U study, Phys. Rev. Lett. 98, 115503 (2007).

[24] Z. Zhong, P. X. Xu and P. J. Kelly, Polarity-induced oxygen vacancies at $\mathrm{LaAlO}_{3} \mathrm{SrTiO}_{3}$ interfaces, Phys. Rev. B 82, 165127 (2010), arXiv:1006.5146. 
[25] P. Hansmann, R. Arita, A. Toschi, S. Sakai, G. Sangiovanni and K. Held, Dichotomy between large local and small ordered magnetic moments in iron-based superconductors, Phys. Rev. Lett. 104, 197002 (2010), arXiv:1003.2162.

[26] A. Galler, C. Taranto, M. Wallerberger, M. Kaltak, G. Kresse, G. Sangiovanni, A. Toschi and K. Held, Screened moments and absence of ferromagnetism in FeAl, Phys. Rev. B 92, 205132 (2015), arXiv:1506.00908.

[27] T. Maier, M. Jarrell, T. Pruschke and M. H. Hettler, Quantum cluster theories, Rev. Mod. Phys. 77, 1027 (2005), arXiv:cond-mat/0404055.

[28] H. Kusunose, Influence of spatial correlations in strongly correlated electron systems: Extension to dynamical mean field approximation, J. Phys. Soc. Jpn. 75, 054713 (2006), arXiv:cond-mat/0602451.

[29] A. Toschi, A. A. Katanin and K. Held, Dynamical vertex approximation: A step beyond dynamical mean-field theory, Phys. Rev. B 75, 045118 (2007), arXiv:cond-mat/0603100 .

[30] A. N. Rubtsov, M. I. Katsnelson and A. I. Lichtenstein, Dual fermion approach to nonlocal correlations in the Hubbard model, Phys. Rev. B 77, 033101 (2008).

[31] C. Slezak, J. M., T. Maier and J. Deisz, Multi-scale extensions to quantum cluster methods for strongly correlated electron systems, J. Phys.: Condens. Matter 21, 435604 (2009), arXiv:cond-mat/0603421 .

[32] A. A. Katanin, A. Toschi and K. Held, Comparing pertinent effects of antiferromagnetic fluctuations in the two- and three-dimensional hubbard model, Phys. Rev. B 80, 075104 (2009), arXiv:0808.0689.

[33] A. Sekiyama, H. Fujiwara, S. Imada, S. Suga, H. Eisaki, S. I. Uchida, K. Takegahara, H. Harima, Y. Saitoh, I. A. Nekrasov, G. Keller, D. E. Kondakov, A. V. Kozhevnikov, T. Pruschke, K. Held, D. Vollhardt and V. I. Anisimov, Mutual experimental and theoretical validation of bulk photoemission spectra of $\mathrm{Sr}_{1-x} \mathrm{Ca}_{x} \mathrm{VO}_{3}$, Phys. Rev. Lett. 93, 156402 (2004), arXiv:condmat/0312429.

[34] T. Yoshida, K. Tanaka, H. Yagi, A. Ino, H. Eisaki, A. Fujimori and Z.-X. Shen, Direct observation of the mass renormalization in $\mathrm{SrVO}_{3}$ by angle resolved photoemission spectroscopy, Phys. Rev. Lett. 95, 146404 (2005), arXiv:cond-mat/0504075.

[35] I. H. Inoue, I. Hase, Y. Aiura, A. Fujimori, K. Morikawa, T. Mizokawa, Y. Haruyama, T. Maruyama and Y. Nishihara, Systematic change of spectral function observed by controlling electron correlation in $\mathrm{Ca}_{1 x} \mathrm{Sr}_{x} \mathrm{VO}_{3}$ with fixed $3 d^{1}$ configuration, Physica C 235-240, 1007 (1994).

[36] K. Yoshimatsu, T. Okabe, H. Kumigashira, S. Okamoto, S. Aizaki, A. Fujimori and M. Oshima, Dimensional-crossover-driven metal-insulator transition in $\mathrm{SrVO}_{3}$ ultrathin films, Phys. Rev. Lett. 104, 147601 (2010).

[37] E. R. Ylvisaker, W. E. Pickett and K. Koepernik, Anisotropy and magnetism in the LSDA+ $U$ method, Phys. Rev. B 79, 035103 (2009), arXiv:0808.1706. 
[38] A. G. Petukhov, I. I. Mazin, L. Chioncel and A. I. Lichtenstein, Correlated metals and the LDA $+U$ method, Phys. Rev. B 67, 153106 (2003), arXiv:condmat/0206548.

[39] F. Lechermann, A. Georges, A. Poteryaev, S. Biermann, M. Posternak, A. Yamasaki and O. K. Andersen, Dynamical mean-field theory using Wannier functions: A flexible route to electronic structure calculations of strongly correlated materials, Phys. Rev. B 74, 125120 (2006), arXiv:cond-mat/0605539.

[40] O. Grånäs, I. D. Marco, P. Thunström, L. Nordström, O. Eriksson, T. Björkman and J. M. Wills, Charge self-consistent dynamical mean-field theory based on the full-potential linear muffin-tin orbital method: Methodology and applications, Comput. Mater. Sci. 55, 295 (2012), arXiv:1110.2606.

[41] H. Park, A. J. Millis and C. A. Marianetti, Computing total energies in complex materials using charge self-consistent DFT+DMFT, Phys. Rev. B 90, 235103 (2014), arXiv:1409.4135.

[42] I. Leonov, V. I. Anisimov and D. Vollhardt, First-principles calculation of atomic forces and structural distortions in strongly correlated materials, Phys. Rev. Lett. 112, 146401 (2014), arXiv:1311.4493.

[43] K. Lejaeghere, G. Bihlmayer, T. Björkman, P. Blaha, S. Blügel, V. Blum, D. Caliste, I. E. Castelli, S. J. Clark, A. Dal Corso, S. de Gironcoli, T. Deutsch, J. K. Dewhurst, I. Di Marco, C. Draxl, M. Dułak, O. Eriksson, J. A. Flores-Livas, K. F. Garrity, L. Genovese, P. Giannozzi, M. Giantomassi, S. Goedecker, X. Gonze, O. Grånäs, E. K. U. Gross, A. Gulans, F. Gygi, D. R. Hamann, P. J. Hasnip, N. A. W. Holzwarth, D. Iuşan, D. B. Jochym, F. Jollet, D. Jones, G. Kresse, K. Koepernik, E. Küçükbenli, Y. O. Kvashnin, I. L. M. Locht, S. Lubeck, M. Marsman, N. Marzari, U. Nitzsche, L. Nordström, T. Ozaki, L. Paulatto, C. J. Pickard, W. Poelmans, M. I. J. Probert, K. Refson, M. Richter, G.-M. Rignanese, S. Saha, M. Scheffler, M. Schlipf, K. Schwarz, S. Sharma, F. Tavazza, P. Thunström, A. Tkatchenko, M. Torrent, D. Vanderbilt, M. J. van Setten, V. Van Speybroeck, J. M. Wills, J. R. Yates, G.-X. Zhang and S. Cottenier, Reproducibility in density functional theory calculations of solids, Science 351, 1415 (2016).

[44] K. Schwarz, P. Blaha and G. Madsen, Electronic structure calculations of solids using the WIEN2k package for material sciences, Comp. Phys. Commun. 147, 71 (2002).

[45] W. Kohn and L. J. Sham, Self-consistent equations including exchange and correlation effects, Phys. Rev. 140, A1133 (1965).

[46] J. P. Perdew and Y. Wang, Accurate and simple analytic representation of the electron-gas correlation energy, Phys. Rev. B 45, 13244 (1992).

[47] J. P. Perdew, K. Burke and M. Ernzerhof, Generalized gradient approximation made simple, Phys. Rev. Lett. 77, 3865 (1996).

[48] Z. Zhong, P. Wissgott, K. Held and G. Sangiovanni, Microscopic understanding of the orbital splitting and its tuning at oxide interfaces, EPL 99, 37011 (2012), arXiv:1205.4001 . 
[49] N. Marzari and D. Vanderbilt, Maximally localized generalized Wannier functions for composite energy bands, Phys. Rev. B 56, 12847 (1997), arXiv:cond-mat/9707145.

[50] N. Marzari, I. Souza and D. Vanderbilt, An introduction to maximallylocalized Wannier functions, in $\Psi_{k}$ Newsletter (Highlight 57) (2003).

[51] N. Marzari, A. A. Mostofi, J. R. Yates, I. Souza and D. Vanderbilt, Maximally localized Wannier functions: Theory and applications, Rev. Mod. Phys. 84, 1419 (2012), arXiv:1112.5411.

[52] A. A. Mostofi, J. R. Yates, Y.-S. Lee, I. Souza, D. Vanderbilt and N. Marzari, wannier90: A tool for obtaining maximally-localised Wannier functions, Comp. Phys. Commun. 178, 685 (2008), arXiv:0708.0650.

[53] J. Kuneš, R. Arita, P. Wissgott, A. Toschi, H. Ikeda and K. Held, Wien2wannier: From linearized augmented plane waves to maximally localized Wannier functions, Comput. Phys. Commun. 181, 1888 (2010), arXiv:1004.3934.

[54] I. A. Nekrasov, K. Held, G. Keller, D. E. Kondakov, T. Pruschke, M. Kollar, O. K. Andersen, V. I. Anisimov and D. Vollhardt, Momentum-resolved spectral functions of $\mathrm{SrVO}_{3}$ calculated by LDA+DMFT, Phys. Rev. B 73, 155112 (2006), arXiv:cond-mat/0508313.

[55] E. Gull, A. J. Millis, A. I. Lichtenstein, A. N. Rubtsov, M. Troyer and P. Werner, Continuous-time Monte Carlo methods for quantum impurity models, Rev. Mod. Phys. 83, 349 (2011), arXiv:1012.4474.

[56] N. Parragh, A. Toschi, K. Held and G. Sangiovanni, Conserved quantities of $S U$ (2)-invariant interactions for correlated fermions and the advantages for quantum Monte Carlo simulations, Phys. Rev. B 86, 155158 (2012), arXiv:1209.0915.

[57] M. Jarrell and J. Gubernatis, Bayesian inference and the analytic continuation of imaginary-time quantum Monte Carlo data, Phys. Rep. 269, 133 (1996).

[58] G. Keller, K. Held, V. Eyert, D. Vollhardt and V. I. Anisimov, Electronic structure of paramagnetic $\mathrm{v}_{2} \mathrm{O}_{3}$ : Strongly correlated metallic and mott insulating phase, Phys. Rev. B 70, 205116 (2004), arXiv:cond-mat/0402133.

[59] R. Hesper, L. H. Tjeng, A. Heeres and G. A. Sawatzky, Photoemission evidence of electronic stabilization of polar surfaces in $\mathrm{K}_{3} \mathrm{C}_{60}$, Phys. Rev. B 62, 16046 (2000).

[60] S. Okamoto and A. J. Millis, Electronic reconstruction at an interface between a Mott insulator and a band insulator, Nature (London) 428, 630 (2004), arXiv:cond-mat/0507150 .

[61] S. Okamoto, A. J. Millis and N. A. Spaldin, Lattice relaxation in oxide heterostructures: $\mathrm{LaTiO}_{3} / \mathrm{SrTiO}_{3}$ superlattices, Phys. Rev. Lett. 97, 056802 (2006), arXiv:cond-mat/0601081 .

[62] S. Okamoto and A. J. Millis, Spatial inhomogeneity and strong correlation physics: A dynamical mean-field study of a model Mott-insulatorband-insulator heterostructure, Phys. Rev. B 70, 241104 (2004), arXiv:condmat/0407592. 
[63] P. Hansmann, X. Yang, A. Toschi, G. Khaliullin, O. K. Andersen and K. Held, Turning a nickelate Fermi surface into a cupratelike one through heterostructuring, Phys. Rev. Lett. 103, 016401 (2009), arXiv:0807.0407.

[64] F. Lechermann, L. Boehnke and D. Grieger, Formation of orbital-selective electron states in $\mathrm{LaTiO}_{3} / \mathrm{SrTiO}_{3}$ superlattices, Phys. Rev. B 87, 241101 (2013), arXiv:1304.2013.

[65] F. Lechermann, L. Boehnke, D. Grieger and C. Piefke, Electron correlation and magnetism at the $\mathrm{LaAlO}_{3} / \mathrm{SrTiO}_{3}$ interface: A DFT+DMFT investigation, Phys. Rev. B 90, 085125 (2014), arXiv:1401.6105.

[66] F. Lechermann, H. O. Jeschke, A. J. Kim, S. Backes and R. Valentí, Electron dichotomy on the $\mathrm{SrTiO}_{3}$ defect surface augmented by many-body effects, Phys. Rev. B 93, 121103 (2016), arXiv:1603.04986.

[67] M. Altmeyer, H. O. Jeschke, O. Hijano-Cubelos, C. Martins, F. Lechermann, K. Koepernik, A. F. Santander-Syro, M. J. Rozenberg, R. Valentí and M. Gabay, Magnetism, spin texture, and in-gap states: Atomic specialization at the surface of oxygen-deficient $\mathrm{SrTiO}_{3}$, Phys. Rev. Lett. 116, 157203 (2016), arXiv:1511.08614.

[68] E. Pavarini, S. Biermann, A. Poteryaev, A. I. Lichtenstein, A. Georges and O. K. Andersen, Mott transition and suppression of orbital fluctuations in orthorhombic $3 d^{1}$ perovskites, Phys. Rev. Lett. 92, 176403 (2004), arXiv:condmat/0309102.

[69] E. Pavarini, A. Yamasaki, J. Nuss and O. K. Andersen, How chemistry controls electron localization in $3 d^{1}$ perovskites: a Wannier-function study, New J. Phys. 7, 188 (2005), arXiv:cond-mat/0504034.

[70] K. Dymkowski and C. Ederer, Strain-induced insulator-to-metal transition in $\mathrm{LaTiO}_{3}$ within DFT+DMFT, Phys. Rev. B 89, 161109 (2014), arXiv:1311.5130.

[71] F. Lechermann and M. Obermeyer, Towards Mott design by $\delta$-doping of strongly correlated titanates, New J. Phys. 17, 043026 (2015), arXiv:1411.1637.

[72] J. L. García-Muñoz, J. Rodríguez-Carvajal, P. Lacorre and J. B. Torrance, Neutron-diffraction study of $R \mathrm{NiO}_{3}$ ( $R=\mathrm{La}, \mathrm{Pr}, \mathrm{Nd}, \mathrm{Sm}$ ): Electronically induced structural changes across the metal-insulator transition, Phys. Rev. B 46, 4414 (1992).

[73] J. B. Torrance, P. Lacorre, A. I. Nazzal, E. J. Ansaldo and C. Niedermayer, Systematic study of insulator-metal transitions in perovskites $R \mathrm{NiO}_{3}(R=\mathrm{Pr}$, $\mathrm{Nd}, \mathrm{Sm}, \mathrm{Eu})$ due to closing of charge-transfer gap, Phys. Rev. B 45, 8209 (1992).

[74] G. Catalan, Progress in perovskite nickelate research, Phase Transitions 81, 729 (2008).

[75] J. Rodríguez-Carvajal, S. Rosenkranz, M. Medarde, P. Lacorre, M. T. Fernandez-Díaz, F. Fauth and V. Trounov, Neutron-diffraction study of the magnetic and orbital ordering in ${ }^{154} \mathrm{SmNiO}_{3}$ and ${ }^{153} \mathrm{EuNiO}_{3}$, Phys. Rev. B 57, 456 (1998). 
[76] J. A. Alonso, J. L. García-Muñoz, M. T. Fernández-Díaz, M. A. G. Aranda, M. J. Martínez-Lope and M. T. Casais, Charge disproportionation in $\mathrm{RNiO}_{3}$ perovskites: Simultaneous metal-insulator and structural transition in $\mathrm{YNiO}_{3}$, Phys. Rev. Lett. 82, 3871 (1999).

[77] U. Staub, G. I. Meijer, F. Fauth, R. Allenspach, J. G. Bednorz, J. Karpinski, S. M. Kazakov, L. Paolasini and F. d'Acapito, Direct observation of charge order in an epitaxial $\mathrm{NdNiO}_{3}$ film, Phys. Rev. Lett. 88, 126402 (2002).

[78] E. A. Nowadnick, J. P. Ruf, H. Park, P. D. C. King, D. G. Schlom, K. M. Shen and A. J. Millis, Quantifying electronic correlation strength in a complex oxide: A combined DMFT and ARPES study of $\mathrm{LaNiO}_{3}$, Phys. Rev. B 92, 245109 (2015), arXiv:1508.07247.

[79] S. Lee, R. Chen and L. Balents, Landau theory of charge and spin ordering in the nickelates, Phys. Rev. Lett. 106, 016405 (2011).

[80] S. Lee, R. Chen and L. Balents, Metal-insulator transition in a two-band model for the perovskite nickelates, Phys. Rev. B 84, 165119 (2011), arXiv:1107.0724.

[81] B. Lau and A. J. Millis, Theory of the magnetic and metal-insulator transitions in $\mathrm{RNiO}_{3}$ bulk and layered structures, Phys. Rev. Lett. 110, 126404 (2013), arXiv:1210.6693.

[82] V. I. Anisimov, D. Bukhvalov and T. M. Rice, Electronic structure of possible nickelate analogs to the cuprates, Phys. Rev. B 59, 7901 (1999).

[83] J. Chaloupka and G. Khaliullin, Orbital order and possible superconductivity in $\mathrm{LaNiO}_{3} / \mathrm{LaMO}_{3}$ superlattices, Phys. Rev. Lett. 100, 016404 (2008), arXiv:1005.1464.

[84] P. Hansmann, A. Toschi, X. Yang, O. K. Andersen and K. Held, Electronic structure of nickelates: From two-dimensional heterostructures to threedimensional bulk materials, Phys. Rev. B 82, 235123 (2010).

[85] M. J. Han, X. Wang, C. A. Marianetti and A. J. Millis, Dynamical meanfield theory of nickelate superlattices, Phys. Rev. Lett. 107, 206804 (2011), arXiv:1105.0016.

[86] N. Parragh, G. Sangiovanni, P. Hansmann, S. Hummel, K. Held and A. Toschi, Effective crystal field and Fermi surface topology: A comparison of $d$ - and $d p$-orbital models, Phys. Rev. B 88, 195116 (2013), arXiv:1303.2099.

[87] O. E. Peil, M. Ferrero and A. Georges, Orbital polarization in strained $\mathrm{LaNiO}_{3}$ : Structural distortions and correlation effects, Phys. Rev. B 90, 045128 (2014), arXiv:1404.7622.

[88] E. Benckiser, M. W. Haverkort, S. Brück, E. Goering, S. Macke, A. Frañó, X. Yang, O. K. Andersen, G. Cristiani, H.-U. Habermeier, A. V. Boris, I. Zegkinoglou, P. Wochner, H.-J. Kim, V. Hinkov and B. Keimer, Orbital reflectometry of oxide heterostructures, Nat. Mater. 10, 189 (2011), arXiv:1101.0238.

[89] M. Wu, E. Benckiser, M. W. Haverkort, A. Frano, Y. Lu, U. Nwankwo, S. Brück, P. Audehm, E. Goering, S. Macke, V. Hinkov, P. Wochner, G. Christiani, S. Heinze, G. Logvenov, H.-U. Habermeier and B. Keimer, Strain and 
composition dependence of orbital polarization in nickel oxide superlattices, Phys. Rev. B 88, 125124 (2013), arXiv:1308.6389.

[90] A. S. Disa, D. P. Kumah, A. Malashevich, H. Chen, D. A. Arena, E. D. Specht, S. Ismail-Beigi, F. J. Walker and C. H. Ahn, Orbital engineering in symmetry-breaking polar heterostructures, Phys. Rev. Lett. 114, 026801 (2015).

[91] H. Park, A. J. Millis and C. A. Marianetti, Influence of quantum confinement and strain on orbital polarization of four-layer $\mathrm{LaNiO}_{3}$ superlattices: A DFT+DMFT study, Phys. Rev. B 93, 235109 (2016), arXiv:1602.01422.

[92] M. Uchida, K. Ishizaka, P. Hansmann, Y. Kaneko, Y. Ishida, X. Yang, R. Kumai, A. Toschi, Y. Onose, R. Arita, K. Held, O. K. Andersen, S. Shin and Y. Tokura, Pseudogap of metallic layered nickelate $R_{2-x} \mathrm{Sr}_{x} \mathrm{NiO}_{4}(R=\mathrm{Nd}, \mathrm{Ei})$ crystals measured using angle-resolved photoemission spectroscopy, Phys. Rev. Lett. 106, 027001 (2011), arXiv:1012.5516.

[93] H. Chen, H. Park, A. J. Millis and C. A. Marianetti, Charge transfer across transition-metal oxide interfaces: Emergent conductance and electronic structure, Phys. Rev. B 90, 245138 (2014), arXiv:1408.0217.

[94] Z. Fang and N. Nagaosa, Quantum versus Jahn-Teller orbital physics in $\mathrm{YVO}_{3}$ and $\mathrm{LaVO}_{3}$, Phys. Rev. Lett. 93, 176404 (2004), arXiv:condmat/0409299.

[95] M. De Raychaudhury, E. Pavarini and O. K. Andersen, Orbital fluctuations in the different phases of $\mathrm{YVO}_{3}$ and $\mathrm{LaVO}_{3}$, Phys. Rev. Lett. 99, 126402 (2007), arXiv:cond-mat/0702316.

[96] Y. Hotta, T. Susaki and H. Y. Hwang, Polar discontinuity doping of the $\mathrm{LaVO}_{3} / \mathrm{SrTiO}_{3}$ interface, Phys. Rev. Lett. 99, 236805 (2007), arXiv:0710.2174.

[97] E. Assmann, P. Blaha, R. Laskowski, K. Held, S. Okamoto and G. Sangiovanni, Oxide heterostructures for efficient solar cells, Phys. Rev. Lett. 110, 078701 (2013), arXiv:1301.1314.

[98] E. Assmann, Spectral Properties of Strongly Correlated Materials, Ph.D. thesis, TU Wien (2015).

[99] P. Werner, K. Held and M. Eckstein, Role of impact ionization in the thermalization of photoexcited mott insulators, Phys. Rev. B 90, 235102 (2014), arXiv:1408.3425.

[100] L. Wang, Y. Li, A. Bera, C. Ma, F. Jin, K. Yuan, W. Yin, A. David, W. Chen, W. Wu, W. Prellier, S. Wei and T. Wu, Device performance of the Mott insulator $\mathrm{LaVO}_{3}$ as a photovoltaic material, Phys. Rev. Applied 3, 064015 (2015).

[101] M. Nakamura, F. Kagawa, T. Tanigaki, H. S. Park, T. Matsuda, D. Shindo, Y. Tokura and M. Kawasaki, Spontaneous polarization and bulk photovoltaic effect driven by polar discontinuity in $\mathrm{LaFeO}_{3} / \mathrm{SrTiO}_{3}$ heterojunctions, Phys. Rev. Lett. 116, 156801 (2016).

[102] G. Koster, L. Klein, W. Siemons, G. Rijnders, J. S. Dodge, C.-B. Eom, D. H. A. Blank and M. R. Beasley, Structure, physical properties, and applications of $\mathrm{SrRuO}_{3}$ thin films, Rev. Mod. Phys. 84, 253 (2012). 
[103] W. E. Bell and M. Tagami, High-temperature chemistry of the rutheniumosygen system, J. Phys. Chem. 67, 2432 (1963).

[104] D. Toyota, I. Ohkubo, H. Kumigashira, M. Oshima, T. Ohnishi, M. Lippmaa, M. Takizawa, A. Fujimori, K. Ono, M. Kawasaki and H. Koinuma, Thickness-dependent electronic structure of ultrathin $\mathrm{SrRuO}_{3}$ films studied by in situ photoemission spectroscopy, Appl. Phys. Lett. 87, 162508 (2005), arXiv: 1505.05692 .

[105] J. Xia, W. Siemons, G. Koster, M. R. Beasley and A. Kapitulnik, Critical thickness for itinerant ferromagnetism in ultrathin films of $\mathrm{SrRuO}_{3}$, Phys. Rev. B 79, 140407 (2009), arXiv:0811.0384.

[106] J. M. Rondinelli, N. M. Caffrey, S. Sanvito and N. A. Spaldin, Electronic properties of bulk and thin film $\mathrm{SrRuO}_{3}$ : Search for the metal-insulator transition, Phys. Rev. B 78, 155107 (2008), arXiv:0806.1315.

[107] P. Mahadevan, F. Aryasetiawan, A. Janotti and T. Sasaki, Evolution of the electronic structure of a ferromagnetic metal: Case of $\mathrm{SrRuO}_{3}$, Phys. Rev. B 80, 035106 (2009), arXiv:0812.2098.

[108] G. Rijnders, D. H. A. Blank, J. Choi and C.-B. Eom, Enhanced surface diffusion through termination conversion during epitaxial $\mathrm{SrRuO}_{3}$ growth, Appl. Phys. Lett. 84, 505 (2004).

[109] E. Jakobi, S. Kanungo, S. Sarkar, S. Schmitt and T. Saha-Dasgupta, LDA+DMFT study of Ru-based perovskite $\mathrm{SrRuO}_{3}$ and $\mathrm{CaRuO}_{3}$, Phys. Rev. B 83, 041103 (2011).

[110] M. Kim and B. I. Min, Nature of itinerant ferromagnetism of $\mathrm{SrRuO}_{3}$ : A DFT+DMFT study, Phys. Rev. B 91, 205116 (2015).

[111] L. Si, Z. Zhong, J. M. Tomczak and K. Held, Route to room-temperature ferromagnetic ultrathin $\mathrm{SrRuO}_{3}$ films, Phys. Rev. B 92, 041108 (2015), arXiv:1503.00640.

[112] E. Assmann, P. Wissgott, J. Kuneš, A. Toschi, P. Blaha and K. Held, woptic: Optical conductivity with Wannier functions and adaptive k-mesh refinements, Comp. Phys. Commun. 202, 1 (2016), arXiv:1507.04881.

[113] S. Bhandary, E. Assmann, M. Aichhorn and K. Held, Charge self-consistency in density functional theory + dynamical mean field theory: k-space reoccupation and orbital order, Phys. Rev. B 94, 155131 (2016), arXiv:1605.00545.

[114] D. Xiao, W. Zhu, Y. Ran, N. Nagaosa and S. Okamoto, Interface engineering of quantum Hall effects in digital transition metal oxide heterostructures, Nature Commun. 2, 596 (2011), arXiv:1106.4296. 